\title{
3D bioprinting of complex tissues in vitro: state-of-the-art and future perspectives
}

\author{
Yi Xiang ${ }^{1} \cdot$ Kathleen Miller ${ }^{1} \cdot$ Jiaao Guan ${ }^{2} \cdot$ Wisarut Kiratitanaporn ${ }^{1} \cdot$ Min Tang $^{1} \cdot$ Shaochen Chen ${ }^{1,2}$ (I)
}

Received: 15 November 2021 / Accepted: 20 December 2021 / Published online: 10 January 2022

(c) The Author(s) 2022

\begin{abstract}
The pharmacology and toxicology of a broad variety of therapies and chemicals have significantly improved with the aid of the increasing in vitro models of complex human tissues. Offering versatile and precise control over the cell population, extracellular matrix (ECM) deposition, dynamic microenvironment, and sophisticated microarchitecture, which is desired for the in vitro modeling of complex tissues, $3 \mathrm{D}$ bio-printing is a rapidly growing technology to be employed in the field. In this review, we will discuss the recent advancement of printing techniques and bio-ink sources, which have been spurred on by the increasing demand for modeling tactics and have facilitated the development of the refined tissue models as well as the modeling strategies, followed by a state-of-the-art update on the specialized work on cancer, heart, muscle and liver. In the end, the toxicological modeling strategies, substantial challenges, and future perspectives for 3D printed tissue models were explored.
\end{abstract}

Keywords $3 \mathrm{D}$ printing $\cdot$ Tissue model $\cdot$ In vitro model $\cdot$ Toxicity screening $\cdot$ Tissue engineering $\cdot$ Biomaterials

\section{Introduction}

In vitro tissue models have greatly advanced our understanding of the pharmacological and toxicological processes of a wide range of treatments and chemicals (Davila et al. 1998). Such models are efficient, low-cost, and non-cruel recapitulations of native tissues, and their development has sped the discovery of various medications (Madorran et al. 2020), as well as the development of environmental pollution prevention and labor protection approaches. In vitro tissue models have evolved from simple two-dimensional (2D) monocultures into more advanced three-dimensional (3D) structures, such as organoids, dynamic culture systems, micro-tissues, organ-on-chip devices, and other combinations (Braun et al. 2021; Duval et al. 2017). Accurate recapitulation of native physiology, such as cell composition, biophysical and biochemical signaling, as well as microarchitecture, could result in greater substantive response when drawing correlations

Shaochen Chen

chen168@eng.ucsd.edu

1 Department of NanoEngineering, University of California San Diego, La Jolla, USA

2 Department of Electrical and Computer Engineering, University of California San Diego, La Jolla, USA between in vitro and in vivo conditions (Lelièvre et al. 2017).

$3 \mathrm{D}$ bioprinting has emerged as an intriguing approach for the production of complex in vitro models, by which means cells and/or their supporting scaffold are precisely deposited, localized, or joined in user-defined geometries and dimensions. With an ever-expanding range of available biomaterials (Yu et al. 2020a, b) and biocompatible processes (Ashammakhi et al. 2019), 3D bioprinting has aided in the tailored control over microarchitecture, extracellular matrix (ECM) construction, and cell deposition for the establishment of in vitro models, particularly the recapitulation of complex tissues (Ma et al. 2018a, b), and has resulted in significant accomplishments in moving the field forward in recent years.

Here, we present a state-of-the-art review on the in vitro complex tissue model constructions based on 3D bioprinting. We begin with an overview of 3D printing techniques, biomaterials and their use in in vitro tissue construction, and then move on to discussing pioneering work in cancer, heart, liver, and muscle in vitro models for biological studies, drug screening, and toxicity investigations. In the concluding section, we also explore the applications, challenges, and future perspectives of 3D bioprinting technologies and tissue modeling. 


\section{D bioprinting technologies and biomaterials}

\section{D bioprinting technology}

3D bioprinting refers to a type of additive manufacturing, specifically a layer-by-layer fabrication technique that was originally born out of a need for rapid prototyping and has since enjoyed advancement into a fast, customizable fabrication method across many fields. 3D bioprinting technology allows for flexibility in both material choice and design paradigm - in the context of tissue engineering, the ability to incorporate biomaterials and cells inherently allows for 3D bioprinting. As 3D bioprinting becomes more ubiquitous, more research into bioprinting techniques has emerged, allowing for the fabrication of a wide range of biocompatible constructs, and cell-encapsulated tissues, and organ models.

\section{Inkjet-based}

A typical inkjet-based bio-printer is shown in Fig. 1A (Patel 2016). It dispenses droplets of low-viscosity bio-ink from a 'printhead' containing arrays of small nozzle apertures to form patterns and then stabilizes the structure by photocrosslinking or thermal gelation (Yu et al. 2020a, b). Typical inkjet printer designs include: a bioink storage chamber(s), actuators to both guide bioink(s) to the nozzle and form the droplets, and stage/control systems for three-axial movement. There are three main categories of inkjet bioprinting methods: continuous-inkjet, droplet-on-demand, and electro-hydrodynamic jet bioprinting, all of which differ in their method of bioink droplet deployment control. The continuous-inkjet method extrudes streams of bioink droplets, which lack precise droplet control. The droplet-on-demand bioprinter improves droplet control by creating individual droplets at required times by pressurizing the bioink storage chamber, using thermal, piezoelectric, or electrostatic-based actuators. The electro-hydrodynamic jet bioprinter generates droplets by pulling the bio-ink through the nozzle instead
A
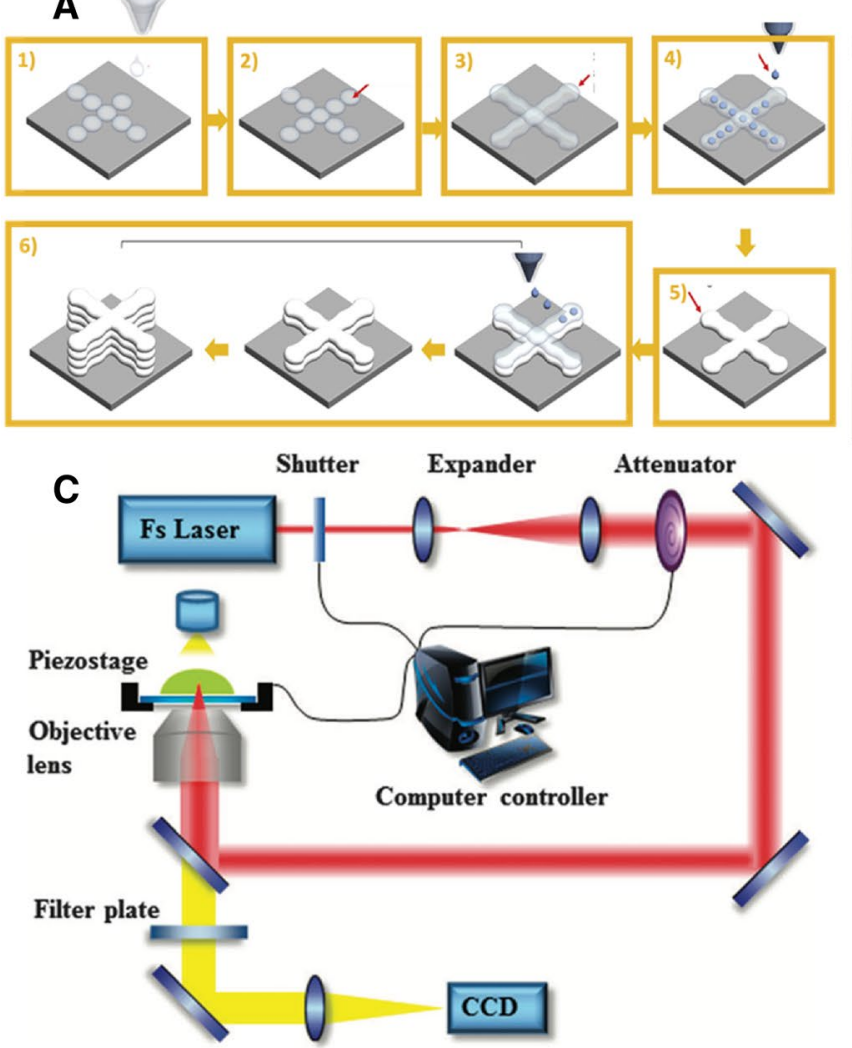

Fig. 1 Schematic illustrations of 3D bioprinting processes. A Inkjetbased (Gudapati et al. 2016): (1) hydrogel precursor droplet deposition on a substrate; (2) droplet spreading; (3) droplets assembling into lines; (4) cross-linker droplet deposition; (5) hydrogel crosslinking;

\section{B}

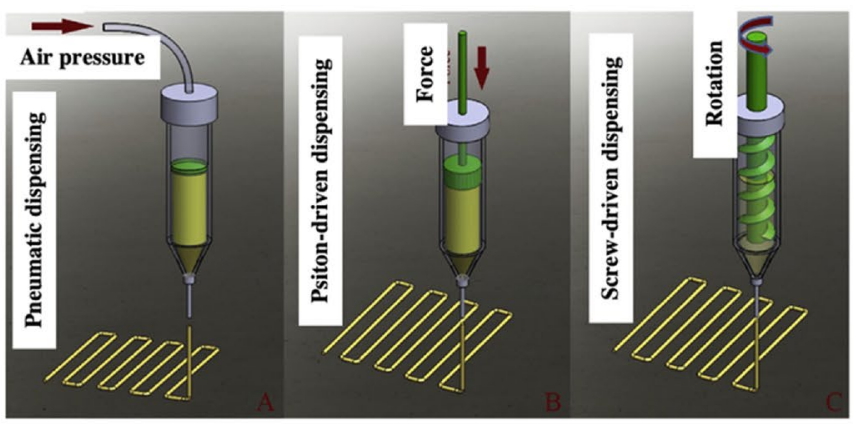

D

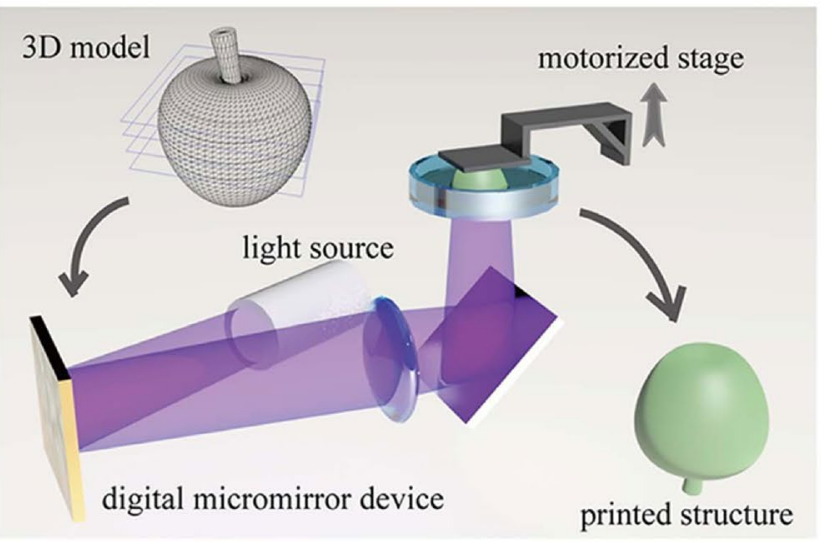

(6) process repeated for layer-by-layer fabrication; B extrusion-based (Derakhshanfar et al. 2018); C TPP-based (Xing et al. 2015); D DLPbased (You et al. 2020) 
of a pushing method with droplet-on-demand bioprinters (Derakhshanfar et al. 2018; Gudapati et al. 2016).

In general, inkjet-based 3D bioprinting has the benefit of precise material deposition with a reasonable printing speed. The utilization of bioink droplets results in minimal material waste and low cost, with a high cell viability (except in thermal inkjet bioprinting). The bioink chamber and nozzle design also allow efficient material replacement and multi-material printing. However, a drawback to inkjet printing is that small nozzle apertures place a lower limit on cell densities in the bioinks-higher cell densities can cause nozzle clogging. Additionally, the low viscosity required for printing may result in limited mechanical strength in the final printed structure. Furthermore, the droplet form of the material causes a limited printing resolution (Gudapati et al. 2016; Mobaraki et al. 2020).

\section{Extrusion-based}

Extrusion-based bioprinting is another widely used bioprinting technology (Fig. 1B). Similar to inkjet printing, extrusion-based printing also extrudes material from a nozzle printhead, but instead of dispensing individual droplets, a typical extrusion printer extrudes a continuous flow of viscous bioink filament. The bioink viscosity used in extrusion bioprinting ranges from 30 to $60 \mathrm{kPa}$ s (Derakhshanfar et al. 2018; Mobaraki et al. 2020). Higher-viscosity bioinks allow for the use of correspondingly higher viscous biomaterials and higher-density cell encapsulation, both of which are beneficial for tissue and organ fabrication. Extrusionbased bioprinter resolutions are limited by the size of the nozzle aperture and material characteristics, and also experience slower printing speeds due to the scanning nature of its printing movement. For both the extrusion-based and inkjet-based bioprinting, supporting structures are needed if creating overhanging 3D structures, which may introduce longer printing times as well as material/cell wastage. The overall fabrication time will vary based on the complexity of the 3D structure (Yu et al. 2020a, b).

\section{Laser polymerization-based}

Laser-based bioprinting methods feature the use of a precisely controlled, focused laser beam to effect patterned photopolymerization in a prepolymer solution. Among them, two-photon polymerization (TPP) bioprinting uses femtosecond laser pulses to achieve submicron level precise printing (Fig. 1C) (Claeyssens et al. 2009; Zhang and Chen 2011; Xing et al. 2015). The high precision afforded by TPP printing allows for the production of micro- and nano-scale tissue scaffolds and vasculatures. However, to ensure precise synchronization between the motion and laser pulses, the TPP printing speed is often limited, and thus requires long fabrication times for complex and/or large structures (Yu et al. 2020a, b). After printing, the un-polymerized residual solution will need to be removed to reveal the printed structure, which causes material waste; however, this material may function as a soft, supportive structure during the actual printing process, thus eliminates the need for designated supporting structures (Yu et al. 2020a, b). TPP printing allows for a variety of material viscosities, which is beneficial for the varied conditions of tissue and organ printing, but higher laser powers can cause thermal damage to cells, resulting in reduced cell viability (Derakhshanfar et al. 2018; Hopp 2012).

\section{Digital light processing (DLP)-based}

DLP-based bioprinting is an emerging photopolymerizationbased bioprinting technique that addresses the primary limitations of previous bioprinting methods-speed and spatial resolution for tissue and organ fabrication. Compared to other methods that require point-by-point or line-by-line scanning to create an individual layer, DLP-based bioprinting drastically reduces the printing time by enabling the projection of an entire 2D design plane at once. A DLP printer's core piece of hardware is a digital micro-mirror array device (DMD), a programmable micro-opto-electromechanical chip with an array of micro-mirrors-this can be used to spatially pattern an incoming light source, such as a $365 \mathrm{~nm}$ ultraviolet or $405 \mathrm{~nm}$ visible light, to photo-polymerize a vat of prepolymer solution (Fig. 1D). The XY resolution of the printed structure is defined by the projection from an individual micro-mirror on the DMD chip, which may be as low as 3-5 $\mu \mathrm{m}$ (and can vary based on intermediate optics and prepolymer characteristics) (Lu et al. 2006), with a typical layer-by-layer Z-resolution limited by the material refill process between subsequent layers (Yu et al. 2020a, b). The Chen group later developed a dynamic optical projection stereolithography (DOPsL) to continuously move the $Z$-axis during printing, resulting in smooth side walls for printed structures (Zhang et al. 2012), and applied this for pre-vascularized tissue printing (Zhu et al. 2017). Further additions to printing efficiency have been researched as well, to go beyond layer-by-layer into volumetric additive manufacturing, where the entire volume is printed at once. One approach is the holographic volumetric 3D fabrication system, which uses three orthogonal light beams with phase-only patterns to print the entire 3D structure in a single step (Shusteff et al. 2017). Another example is the computed axial lithography (CAL) technology, which utilized a DLP projector to illuminate their prepolymer vat from a single side, axially rotating the vat while modulating the projected light's phase pattern (Kelly et al. 2019). Such volumetric additive manufacturing fabrication techniques 
are not limited in printing time by the $Z$-axis, but spatial resolution can suffer due to limitations in holographic precision. Recent efforts by Saha et al. (2019) to address these spatial resolution limitations utilize femtosecond projection two-photon lithography (FP-TPL), which combines the high precision afforded by TPP with the high-throughput nature of DLP-based printing, allowing simultaneous spatial and temporal focusing.

Overall, DLP-based bioprinting enables rapid micro-scale bio-fabrication by plane-wise or even volume-wise printing with the use of a DMD. The high-throughput nature of the process is greatly beneficial for the fabrication of tissues and organs, taking into consideration the time-sensitive nature of live cells and tissues. Additionally, the micro-scale precision afforded by DLP-based printing is similarly beneficial for producing the fine features of native physiology, such as multi-tissue compartments and microvasculature. The relatively low power of the light sources and exposure times typically used also ensure cell viability (Ruskowitz and Deforest 2019). One drawback to DLP-based bioprinting is its pre-filling of a vet of bioink which if not used and recycled, would go to biological waste.

\section{Biomaterials in 3D printing}

Biomaterials form the basis of the matrix or substrate of the final printed structure, and are critical in the in vitro modeling of complex tissues - they provide crucial physical and chemical signals, and can have significant impacts on cell activities, such as adhesion, metabolism, proliferation, differentiation, and migration. For example, the stiffness of the extracellular matrix as a biophysical cue has been found to be deterministic in the viability, differentiation, and migration of a variety of cell types (Gasiorowski et al. 2013). Material porosity crucially aids in the transfer of substances, cell proliferation, and migration ( $\mathrm{Li}$ et al. 2021). The texture of the substrate surface, such as bio-macromolecule alignment and micro/nanostructure, influences adhesion, migration, and maturation, having a substantial impact on tissue organization, remodeling, and development (Fleszar et al. 2018; Liu et al. 2020). Meanwhile, biochemical stimuli are conveyed via the material's backbone or network. The presence of cell adhesion motifs, such as RGD and GFOGER, for example, is crucial for the modeling with adherent cells. Bioactive small molecules, such as metal ions (Yang et al. 2021) and protein imitating peptides (Liu et al. 2010), can be integrated into the material to trigger specific biochemical signaling pathways for specific purposes. Therefore, in addition to taking into account the printing technique, the biomaterials used for in vitro tissue modeling via 3D printing must be carefully chosen based on the tissue of interest, as well as the study's purpose and scientific inquiry. Here, we review the most commonly used biomaterials for 3D bioprinting, with a focus on their characteristics and applications.

\section{Gelatin}

From a chemistry perspective, gelatin is a polypeptide, generated from the hydrolysis of collagen. Gelatin has become one of the most widely used materials in tissue engineering, due to the abundance of cell-adherent RGD motifs in its backbone, excellent biocompatibility, good biodegradability, and low immunogenicity. Its moderate translucency, viscoelasticity and strength also make it appropriate for various bio-fabrication and bioprinting methods. GelMA, a gelatinbased biomaterial in which the primary amines in the lysine backbone of gelatin are replaced with methacrylate groups to facilitate photo-initiated free-radical polymerization, is one of the most common (van Hoorick et al. 2019). For example, GelMA-based micro-constructs with encapsulated conjunctival stem cells were created using DLP-based bioprinting (Zhong et al. 2021a, b), where the GelMA provided a nurturing 3D environment that maintained stem cell phenotype and differentiation potency while maintaining the vitality. Click chemistry methods have been emerging as a way to modify gelatin because of its high efficiency, high selectivity, and minimal side reactions at mild reaction conditions. Gelatin, for example, was modified with norbornene and thiol to allow for photo-reactive thiol-ene crosslinking ( $\mathrm{Yu}$ et al. 2020a, b). The highly selective reaction enabled highorder programmable bio-functionalization and tailored regulation of the mechanical properties of the hydrogel matrix. Click-crosslinked gelatin can also be obtained through the Diels-Alder reaction (García-Astrain et al. 2014) and the carbodiimide reaction (Cammarata et al. 2015).

\section{Hyaluronic acid}

Hyaluronic acid (HA) is a glycosaminoglycan composed of D-glucuronic acid and $\mathrm{N}$-acetyl-D-glucosamine units. The abundance of reactive groups in its backbone allowed various crosslinking and chemical modification strategies to accommodate it for different printing methods. For example, hyaluronic acid glycidyl methacrylate (HAGM) has been synthesized for photo-assisted 3D printing (Liu et al. 2020). Furthermore, adamantane and beta-cyclodextrin were linked to HA to enable a non-covalent guest-host assembly, which introduces shear-thinning behavior into the hydrogel and aids the extrusion-based printing by adding a temporary mechanical support in the printed structure (Ouyang et al. 2016). In native physiology, HA is a key component of the ECM in cartilage, the eyeball, the brain, and a variety of other tissues, as well as a participant in tissue microenvironment, cell signaling, and tumor progression. Thus, in certain cases, HA is required for in vitro modeling of 
complicated tissue. To capture the HA-rich ECM in cartilage tissue, for example, methacrylated HA was introduced to the bioink as a matrix for in vitro chondrogenesis, resulting in enhanced tissue organization (Mouser et al. 2020). In another example, HAGM was used to print the scaffold to support the quiescence state of the limbal stem/progenitor cells (LSC), while the cells remained active when encapsulated in GelMA. The distinct states of the cells in different biomaterials enabled the fabrication of dual-state cells in a single construct, addressing a better mimicry of the native LSC niche (Zhong et al. 2021a, b). For further applications, HA can be modified for click chemistry crosslinking via a variety of mechanisms.

\section{De-cellularized extracellular matrix (dECM)}

De-cellularized extracellular matrix (dECM) of the tissue of interest can be manufactured and applied for 3D printing for enhanced recapitulation of native physiological microenvironments. dECM is not only made up of biopolymers like collagen, fibrin, and glycosaminoglycans as a supporting framework, but also retains the host tissue's native biochemical signaling molecules. While dECM's thermal gelling nature makes it suitable for extrusion-based bioprinting, its poor mechanical strength makes it difficult to use as a bioscaffold on its own - as a result, substantial efforts have been made to composite dECM with other materials. For DLP printing of an in vitro liver lobule tissue, liver dECM was produced and combined 1:1 with GelMA. With the complex biochemical cues given, a stable physiological-mimicking environment for HepG2 3D culture was achieved (Ma et al. 2018a, b).

\section{Alginate}

Alginate is a brown algae-derived copolymer comprising beta-D-mannuronate and alpha-L-guluronate. Alginate is a popular choice for complex in vitro tissue modeling due to its simultaneous nature of high biocompatibility and bioinertness. As a result, alginate makes a good choice for investigations that require minimal degradation as well as precise control of scaffold/substrate stiffness. Alginate possesses the appropriate rheological properties and ion cross-linking capabilities for inkjet and extrusion printing, and can also be chemically modified using 2-aminoethyl methacrylate hydrochloride (AEMA) for photo-assisted printing methods for a broader applicability. Alginate can also build an interpenetrating polymer network (IPN) with other hydrogel materials like collagen and dextran to provide even more customized stiffness and multiplexed bioactivity. For example, alginate was incorporated with collagen to generate the IPN with a tunable storage modulus of 49-419 Pa, the variation of which triggered a reversible state of cancer-associated fibroblast between inflammatory state and myofibroblastic state (Cao et al. 2021).

\section{Synthetic polymers}

Synthetic polymers, in contrast to biopolymers formed from natural sources, are of tremendous interest due to the inherent consistency and feature tailorability that come with scalable industrial manufacturing. One example is polyethylene glycol (PEG), a synthetic polymer extensively used in tissue engineering applications due to its biocompatibility, nonimmunogenic degradation and high capacity for modification. Many functional groups, such as protein-mimicking peptides, growth factors, signaling molecules, and significantly, acrylate groups for photo-polymerization, can be added due to the abundance of reactive hydroxyl groups in the backbone. The mechanical properties of a PEG-based hydrogel, on the other hand, are determined by its molecular weight, the concentration and density of crosslinking synergistically, implying that the substrate's stiffness can be customized by demand. The Young's modulus of PEG diacrylate (PEGDA) ranges from $668 \mathrm{~Pa}$ to $2 \mathrm{kPa}$ as the molecular weight varies from 575 to 20,000 (Vannozzi et al. 2018). Polymers made from various monomers could be used for specific requirements in the substrate or scaffold for in vitro complicated tissue modeling. For example, poly (glycerolco-sebacate), polyurethane, or polycaprolactone can be used to form an elastic substrate, collectively providing a varied range of elastic strength and degradation profile.

\section{State-of-the-art of in vitro tissue models}

Along with the evolution of 3D bioprinting techniques and biomaterials, in vitro models of a variety of tissues, organs and diseases have been developed, evaluated, optimized and brought into use. Here, taking cancer, heart, liver and muscle as examples, we review the pioneering and representative work in the field.

\section{Cancer}

Cancer remains a significant public health issue worldwide due to its high occurrence, mortality, and economic impact. 3D bioprinting offers an opportunity to advance the in vitro modeling of various cancers types, such as brain cancer (Tang et al. 2020, 2021), pancreatic cancer (Hakobyan et al. 2020), liver cancer (Ma et al. 2018a, b), lung cancer, colorectal cancer, ovarian cancer (Xu et al. 2011), breast cancer (Hribar et al. 2015; Zhu et al. 2016), and metastatic models (Meng et al. 2019; Zhu et al. 2016), owing to its ability to recapitulate the complex cellular and material heterogeneities. 


\section{Brain cancer}

Tang et al. developed multicellular glioblastoma (GBM) models using DLP-based bioprinting (Tang et al. 2020). Patient-derived GBM stem cells (GSCs), macrophages, neural progenitor cells, and astrocytes were fabricated into a defined spatial organization to form a brain tumor within brain architecture, recapitulating immune interactions and functional dependencies in 3D microenvironment (Fig. 2A). Tumor cells demonstrated higher drug resistance and invasion capacity with the inclusion of macrophages. Extrusion-based bioprinting was also used to generate GBM models composed of GSCs, patient-derived GBM-associated stromal cells, and microglia in an alginate-based hydrogel
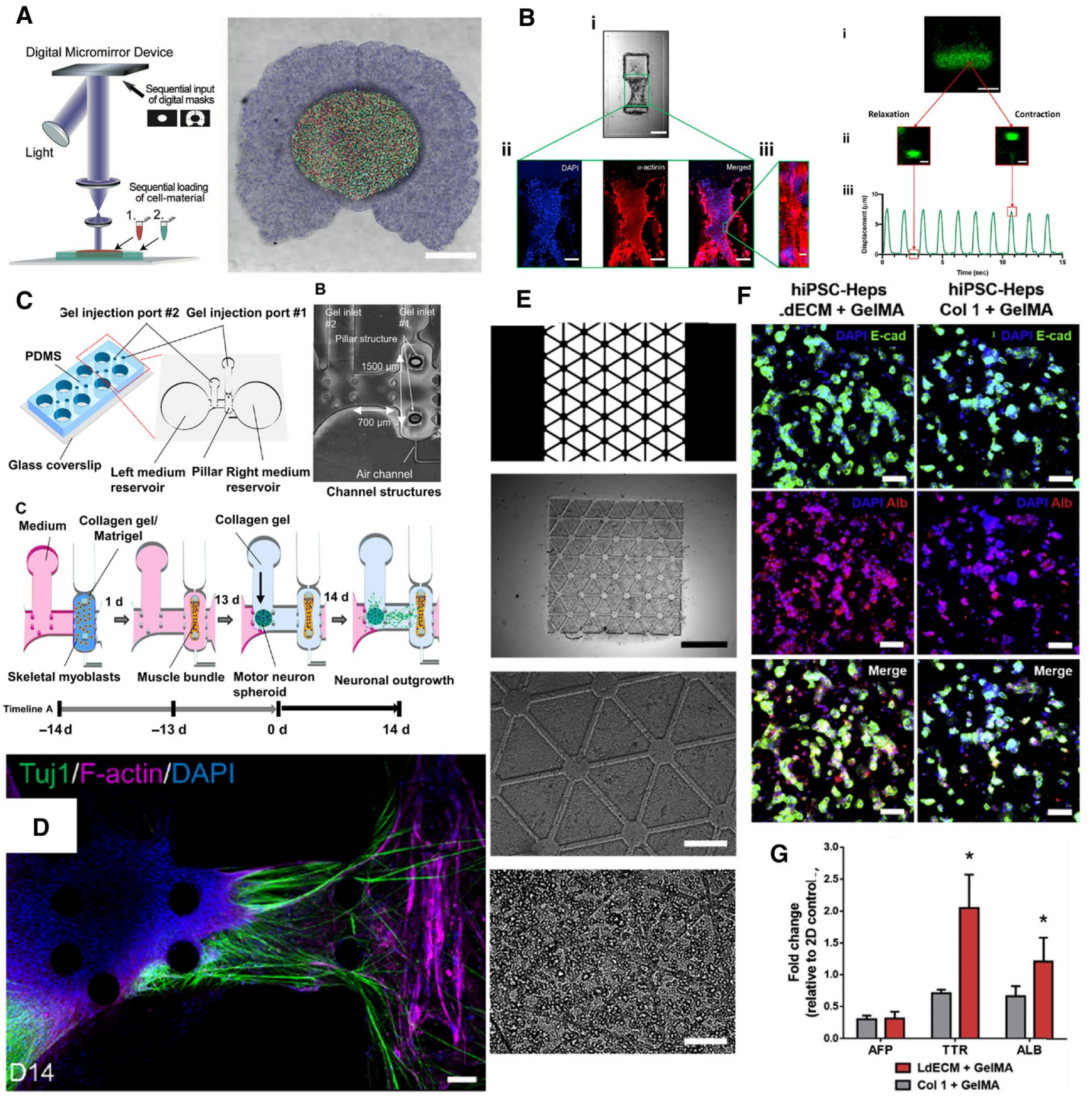

Fig. 2 Examples of recently developed 3D in vitro models. A Fabrication schematic and example print of a GBM model (Tang et al. 2020); B Remodeling, maturation and contraction characterization of a pillar-based heart micro-tissue (Miller et al. 2021) for high-throughput screening; C Illustration of the construction of an ALS model, and D ALS patient-derived motor unit remodeled in the ALS model (Osaki et al. 2018); E High-resolution printing of patient-derived liver model in a ECM-mimetic bioink, and $\mathbf{F}$, $\mathbf{G}$ better remodeling of the phenotype and gene expression profile of iPSC-derived hepatocytes in the ECM-mimetic microenvironment (Yu et al. 2019) 
(Hermida et al. 2020). Alginate hydrogels were modified with RGDs for better cell attachment. The 3D GBM models demonstrated enhanced resistance to cisplatin which failed in many clinical trials but showed promising efficacy in $2 \mathrm{D}$ cell cultures. 3D models have a potential application for more reliable efficacy evaluations.

\section{Pancreatic cancer}

Hakobyan et al. used laser-assisted bioprinting (LAB) for generation of pancreatic ductal adenocarcinoma (PDAC) spheroid arrays consisted of both acinar and ductal cells in GelMA (Hakobyan et al. 2020). These models were used for interrogation of different factors that contribute to the precursor PDAC lesions at the early stage PDAC onset and progression. Xu et al. (2019) developed PDAC models with biomimetic materials consisted of surface-engineered cellulose nanofibrils (CNFs) and photo-crosslinkable galactoglucomannan methacrylates (GGMMAs), and a UVassisted extrusion-based printing techniques. The bioinks demonstrated promising biocompatibility and supported pancreatic cancer cell and dermal fibroblast proliferation. Utama et al. reported a drop-on-demand method to rapidly form PDAC models in 96-well format (Utama et al. 2021). Tunable biological and mechanical properties were enabled by the 4-arm PEG-based polymers that can form hydrogels within seconds.

\section{Lung cancer}

Mondal et al. utilized extrusion-based printing and a sodium alginate-gelatin hydrogel to develop non-small cell lung cancer (NSCLC) co-culture models with patient-derived xenograft cells and cancer-associated fibroblasts (Mondal et al. 2019). The methods demonstrated high printability and good cell viability. After two weeks of in vitro culture, the NSCLC spheroids could reach a diameter ranging from 50 to $1,100 \mu \mathrm{m}$, creating hypoxic cores within the spheroids for further research. Cellular crosstalk created by the co-culture system was confirmed by upregulation of specific genes, such as vimentin and $\alpha$-SMA. Wang et al. reported a method that combined low-temperature molding and 3D bioprinting technique to fabricate a lung cancer model (Wang et al. 2018). A biomimetic 3D hydrogel grid scaffold was generated with gelatin, sodium alginate, and lung cancer cells A549/95-D. Cell proliferation plateaued after two weeks of culture and had a viability over $90 \%$.

\section{Colorectal cancer}

Chen et al. reported a 3D printed colorectal cancer (CRC) model that closely mimicked the physiological functions and cellular crosstalk between the tumor cells and tumor-associated stromal cells (Chen et al. 2020). Co-culture of colorectal cancer cells, cancer-associated fibroblasts, and tumor-associated endothelial cells on bio-printed scaffolds reprogrammed normal stromal cells into tumor-associated phenotypes. Cellular processes and vascularization were observed, and could help elucidate oncogenesis factors and evaluate the efficiency of potential drugs.

Tariq et al. utilized 3D magnetic bioprinted CRC models to investigate $\mathrm{P}$-glycoprotein associated multidrug resistance (MDR) in cancer treatment (Tariq et al. 2020). The authors delivered siRNA, designed against MDR1 gene to silence the gene in Caco- 2 cells and studied the role of MDR- 1 gene in both $2 \mathrm{D}$ and $3 \mathrm{D}$ culture conditions. The 3D model was compared to 2D culture and demonstrated that the knockdown of MDR1 gene in colorectal carcinoma cells can significantly reduce the tumor cell migration in both $2 \mathrm{D}$ cell culture and 3D bioprinted models.

\section{Breast cancer}

Langer et al. (2019) reported generation of a multicellular scaffold-free tumor tissue representing subtypes of breast cancer and pancreatic cancer using 3D printing. The multiple cell types within the printed structure could self-organize into biomimetic morphologies and secreted their own ECMs to reform the tissues. Incorporation of patient-derived cells into the models offeres a translational tool for investigating the therapeutic responses, potential oncogenic endpoints, and crosstalk between different cell types relevant to individual patients.

Vinson et al. (2017) investigated epithelial-adipose interactions in breast cancer using a 3D-printed breast cancer model. Patient-derived breast cancer cells MCF-7 and MDA-MB-231 and differentiated adipocytes were spatially patterned by laser direct-write bioprinting technique. Investigations of early onset of cancer cell invasion through cellular and tissue-level interactions in the adipose tissue were enabled by the 3D models.

Duchamp et al. (2019) established a sacrificial bioprinting strategy to generate biomimetic mammary duct cancer models to study the oncogenesis and invasion processes of breast cancer. The models were first generated with GelMA into duct-like structures, and the channels were then populated with MCF-7 cells, which was reported to have relatively low invasiveness. Agarose was used as a sacrificial material for convenient extraction. The breast cancer model could be cultured over 24 days and outward invasion of cancer cells into the duct-like matrix was observed. This proof-of-concept model demonstrates the potential value of $3 \mathrm{D}$ printed models in studying the mechanism of oncogenesis of breast cancer. 


\section{Heart}

According to a study by the American Heart Association (2021), cardiovascular disease (CVD) affects nearly 50\% of the US population and accounted for more than 360 billion dollars in consumer costs from 2016 to 2017, thus making CVD of significant medical, scientific, and economic importance. To help delve into and improve research surrounding CVD, significant effort has been invested into state-of-theart solutions, from 3D-printed cardiac patches for damaged hearts to engineered heart tissues (EHTs) for evaluating drug efficacy and toxicity (Dvir et al. 2011).

When evaluating a drug, researchers have commonly used traditional 2D cultures and/or animal models. Due to the lack of chemical and biophysical cues a 2D culture receives from its environment; specifically the cell-extracellular matrix, cell-cell, and tissue-level interactions; these cells are unable to properly recapitulate the response of a mature adult heart (Veldhuizen et al. 2019; Zuppinger 2019). On the other end of the spectrum, murine models are appealing as they can capture these environmental cues. However, inter-species differences in ion channels, biological pathways and pharmacokinetic properties negatively impact the predictive ability of these models for human hearts (Mathur et al. 2016). The need for a better predictive model of drug toxicity is paramount, as evidenced by the fact that $45 \%$ of post-approval drug withdrawal from the market is related to cardiovascular system issues (Ferri et al. 2013).

To address these issues, researchers have developed various cardiac models and EHTs. Using a variety of methods, researchers have formed tissues with measurable functionality (beating frequency and force) against drugs or toxins (Mathur et al. 2016; Nam et al. 2015; Veldhuizen et al. 2019). One such EHT is the flexible cardiac thin film, developed by the Parker group (Grosberg et al. 2011). The model was created by seeding cardiac cells onto a thin sheet of fibronectin-stamped polydimethylsiloxane (PDMS), with the stamped fibronectin encouraging an anisotropic cardiac orientation, which is important for optimal force generation. The contractility of the tissue was then evaluated by measuring the "curl" of the thin film that occurred during tissue contraction. After developing this initial model, the group then expanded on the original study with multiple derivations and improvements of the original model (Ahn et al. 2018; Lind et al. 2017; McCain et al. 2013; Wang et al. 2014). In one compelling study, the group investigated Barth syndrome, a cardiomyopathic disease caused by mutated TAZ (the gene Tafazzin), in Cas9-edited induced pluripotent stem cell cardiomyocytes (iPSC-CMs). Using the in vitro model to analyze sarcomere assembly, contractile stress generation, and ROS (reactive oxygen species) production differences in the modified iPSC-CM compared to the wild type, the researchers were able to successfully show that both the reintroduction of wild-type TAZ and that suppression of ROS by the mitochondria reversed cardiomyopathic symptoms (Wang et al. 2014).

However, many researchers have created much thicker tissues, as opposed to thin films, to better recapitulate a mature heart. One of the most common EHTs used in the field for thick tissues is a pillar design, where cardiac cells are attached to two anchor points, either pillars or wires (Hinson et al. 2015; Liu et al. 2020; Ma et al. 2019; Miller et al. 2021; Ronaldson-Bouchard et al. 2018; Tiburcy et al. 2020; Williams et al. 2021). These anchors are commonly formed from molded PDMS, which is then immersed in a suspension of cells and extracellular matrix (ECM), naturally forming a 3D tissue over multiple days of culture. Like the thin films, these models have also been specialized for disease types. For example, Hinson et al. investigated the effect of different titin protein mutations, an important protein for sarcomere functionality. Using the 3D pillar model, the researchers were able to examine the impact of different titin variants on tissue contractility (Hinson et al. 2015). In another study, Williams et al. developed an arrhythmic cardiac model by dosing culture models with methyl-beta cyclodextrin (MBCD). The MBCD induced arrhythmic behavior in the tissue, which subsequently lost calcium handling ability and exhibited fibrotic activity. This impacted the function of the tissue even after removal of MBCD from culture (Williams et al. 2021).

However, these thicker EHTs rely on passive tension for the cardiac cells to self-organize into an anisotropic orientation. To directly address this, some researchers have incorporated 3D bioprinting into the model by directly printing encapsulated cardiac cells into small lines stretching between two pillars (Fig. 2B) (Liu et al. 2020; Miller et al. 2021). This is significant, as alignment has been shown to increase the maturity of the iPSC-CMs, which thereby increases the ability of the cells to recapitulate the adult heart during drug and toxin testing (Guo and Pu 2020; Hirt et al. 2014). Moreover, since the cells are directly printed, the tissues can be aligned in a small spatial footprint, enabling high-throughput testing on a 96-well plate, as opposed to the 24- or 48-well format used by other pillar models (Miller et al. 2021).

Applications of 3D printing have also expanded into full heart and chamber models (Lee et al. 2019; Wang et al. 2021). However, the field still has a long way to go before regularly using these models for in vitro testing. In particular, the immaturity of iPSC-CMs, which are the preferred cardiomyocyte source, is a persistent issue (Hirt et al. 2014; Ronaldson-Bouchard et al. 2018). To fully repair or even recapitulate an adult heart, we need to have a cell source that is robust and mature. Nevertheless, 3D models have undoubtedly advanced the field of human cardiac research and will continue to do so as new techniques are developed. 


\section{Liver}

The liver functions natively as the primary site for metabolism and detoxification in the body, thus making it a popular tissue for in vitro modeling of screening drugs, mechanistic studies, and liver regeneration. A wide range of biomaterials, such as gelatin, alginate, GelMA, dECM, hyaluronan, and collagen in different combinations, as well as different cell sources, such as primary hepatocytes, liver cancer cell lines, and stem cell-derived hepatocytes, have been utilized for creating biomimetic structures of liver (Ma et al. 2016).

Kang et al. developed a vascularized liver lobule array model with hepatic cells and endothelial cells using preset extrusion bioprinting (Kang et al. 2020). Briefly, a preset cartridge with hepatic, endothelial, and lumen regions was fabricated and injected with bioink with hepatic cells, endothelial cells, and sacrificial materials, respectively. Spatially patterned models of different cell types demonstrated improved functional properties of liver, including higher albumin secretion and urea production, compared to mixed cell types with no spatial organization. Endothelial cells provided structural integrity of the model after culturing for a week. Grix et al. reported a perfusion-enabled liver model with twelve micro-channels open at both sides in a hexagonal structure (Grix et al. 2018). HepaRG cells and human stellate cells were patterned using stereolithography. Stable expression of tight junctions and metabolism markers was observed in the model. The channels within the bioprinted liver were shown to be perfusable.

Efforts have been made on developing more biomimetic bioink for liver tissue engineering. Several groups reported the benefits of using liver dECM for generating liver models, in terms of improved printability, mechanical properties, as well as biological properties (Kim et al. 2020; Lee et al. 2017; Yu et al. (2019) developed photocrosslinkable liver-specific dECM bioinks to generate complex patient-specific liver models. HiPSC-derived hepatocytes were patterned into hexagonal microscale architectures and demonstrated high cell viability and improved maturation in AECM bioinks compared to collagen bioink (Fig. 2E-G). Mao et al. (2020) also reported using DLP-based bioprinting and GelMA/dECM to generate a micro-liver tissue with improved hepatic function restoration. Human-induced hepatocytes demonstrated improved viability and functionality in bioink with dECM compared to bioink with only GelMA. Mazzocchi et al. (2019) improved the printability of collagen I by mixing it with thiolated hyaluronic acid at various ratios. Primary hepatocytes and stellate cells were printed using the composite bioink which remained viable for two weeks. Gori et al. (2020) developed a thermo-responsive hydrogel with alginate and sacrificial Pluronic materials for culturing hepatic cells in 3D constructs. The Pluronic materials improved the diffusion properties of the hydrogel and supported better cell viability.

Goulart et al. (2020) compared using single cell dispersion, 2D HiPSC-hepatocytes, and HiPSC-hepatocyte spheroids for 3D printing with non-parenchymal cells. Single cell format had the worst performance, demonstrating reduced viability and hepatic functions after 18-day cultivation period. Loss of hepatic phenotype was also observed in single cell models. In contrast, spheroid-based models demonstrated improved functionality and stability.

Engineered human liver models have a steadily increased use in the pharmaceutical industry due to their improved functionality, maturation, and steady metabolism compared to 2D-cultured cells (Underhill and Khetani 2018). The drug acetaminophen demonstrated significantly increased sensitivity in 3D-printed liver models than their 2D counterparts (Gori et al. 2020). The liver models can also serve as tools for viral study and infectious viruses. Bioprinted liver models using optimized bioink consisting of alginate, gelatin, and dECM were efficiently transduced with adenoassociated virus (AAV) and supported adenovirus replication (Hiller et al. 2018).

\section{Muscle}

Skeletal muscle is the most abundant tissue in the human body and is innervated by motor neurons through neuromuscular junctions (NMJs), crucial for both locomotion and the coordination of tasks through directional force generation via contraction and relaxation of myofibers (Grefte et al. 2007). Healthy skeletal muscle tissue possesses the ability to regenerate upon injury through the migration, proliferation, and differentiation of nearby satellite cells into mature functional myofibers (Greising et al. 2019), but can lose this regenerative capacity due to a number of factors, including but not limited to traumatic injury, aging, or diseases, such as amyotrophic lateral sclerosis (ALS), Duchenne muscular dystrophy (DMD), myotonic dystrophy (DM), spinal muscular atrophy, and myasthenia gravis (MG), which can lead to a reduction in quality of life (Larkindale et al. 2014, Cappello and Francolini 2017).

To understand the underlying mechanisms of muscular disorders to come up with novel therapeutic intervention strategies, research efforts have been made toward developing physiologically relevant in vitro skeletal muscle models to overcome the limitations imposed by current animal models, such as species-species pathological differences and response.

Microfluidic device serves as suitable candidates for developing in vitro NMJ platforms, where muscle and motor neuron cells can be cultured in separate chambers with their own media reservoirs, but have a bridge 
connecting them, allowing for axonal sprouting to recapitulate a functional NMJ. Santhanam et al. (2018) developed a microfluidic in vitro NMJ model for drug toxicity testing where human skeletal myoblasts were co-cultured with human motoneurons (MNs) in separate PDMS chambers with a microtunnel array connecting the two chambers as described, allowing for axonal outgrowth. The MNs were electrically stimulated via electrodes, with the resulting myotube contractions exhibiting dose-dependent responses to the toxins-Bungarotoxin, BOTOX ${ }^{\circledR}$, and curare (Santhanam et al. 2018). Osaki et al. utilized a similar setup to develop the first ALS-on-a-chip model by co-culturing iPSC-derived skeletal muscle cells connected via a collagen gel bridge to ALS patient-derived MN spheroids that were opto-genetically engineered to allow for optically stimulated muscle contraction with optical stimulation (Fig. 2C) (Osaki et al. 2018) Their model, measured via micropillar displacement, exhibited ALS pathological features with reduced muscle contraction force (Fig. 2D), that improved upon treatment with ALS drug candidates rapamycin and bosutinib, thus creating a platform for ALS drug screening and disease modeling. Another ALS-on-achip platform with iPSC-derived MNs from ALS patients was used by Guo et al. (2020) to investigate NMJ functionality in ALS lines with mutated genes (Guo et al. 2020). Using the same opto-genetic system as Osaki et al. Vila et al. (2019) developed an in vitro microfluidic model to characterize impaired NMJ in MG by co-culturing human skeletal myoblasts and optogenetically engineered neurospheres derived from the same donor, creating the first patient-specific human NMJ (hNMJ). The group recapitulated diseased NMJ pathology by subjecting their NMJ model to serum derived from MG patients and observing diminished NMJ function, as skeletal muscle cells failed to contract upon optical stimulation of MNs. Building upon this platform, the same group incorporated automated video-processing algorithms to create a diagnostic tool that automatically quantifies NMJ function and MG severity in a high-throughput manner based on sera from MG donors (Vila et al. 2021). Another opto-genetically engineered NMJ model was recently developed by Solomon et al. to test the functionality of NMJ in response to vecuronium, a competitive inhibitor of acetylcholine receptors (AChR) (Solomon et al. 2021). The first mature hNMJ model was observed by Bakooshli where primary human myoblasts and human pluripotent stem cell (hPSC)-derived MNs were directed to self-assemble in $3 \mathrm{D}$ co-culture, modeling NMJ function in healthy versus MG-afflicted conditions. Mature functional NMJs were observed generating calcium transients in response to glutamate stimuli with and without MG patient-derived IgG treatments. Brack et al.'s (2019) model was the first in vitro hNMJ model to demonstrate the upregulated expression of adult $\mathrm{AChR}$ epsilon in muscle fibers, thus creating a mature hNMJ platform to study pathology and development of diseases that affect adult NMJs. A recent study led by Faustino Martins et al. created a breakthrough in generating self-assembled 3D human neuromuscular organoids (NMOs) containing supporting terminal Schwann cells and functional NMJs capable of modeling MG pathology through IgG treatment from MG patients. The 3D NMOs were developed from hiPSC-derived neuro-mesodermal progenitors and were capable of being used for long-term culture studies, with the NMJs reported to being stable up to 150 days (Faustino Martins et al. 2020).

In the context of personalized disease modeling, Maffioletti et al. developed an in vitro humanized muscular dystrophy model by generating iPSC-differentiated skeletal muscles derived from patients with DMD, limb-girdle muscular dystrophy, and skeletal laminopathies. Using this 3D platform, abnormal phenotypes of muscular dystrophies were observed, thus providing a platform to study the development of various muscular disorders and potential treatment regimen (Maffioletti et al. 2018).

Recently, Mondrinos et al. (2021) developed an in vitro muscle model where MSC-derived muscle cells were allowed to "sculpt" themselves into muscle tissue via anisotropic contraction post differentiation with functionalized nodes in the PDMS mold serving as "muscle anchors" to prevent detachment. This proof-of-concept model was used to mimic oxidative injury through hydrogen peroxide treatment, and the therapeutic effect of the drugOlaparib and a combination of retinoic acid (RA) and omega-3 fatty acid eicosapentaenoic acid (EPA) pre-treatments was observed to rescue citrate synthase (CS) activity. By adding a tumor compartment containing A549 spheroids, lung fibroblasts, and macrophages into a separate microfluidic chamber, a model of lung cancer cachexia was developed based on this aforementioned oxidative injury model (Mondrinos et al. 2021).

The utilization of 3D nozzle-based bioprinting technology has also been very recently applied to the fabrication of NMJ to recapitulate the complex 3D NMJ structure more closely by spatial cell patterning (Kong et al. 2021; Sanz et al. 2021); however, 3D in vitro NMJ models generated from microfluidic devices still remain a more widely used alternative to accommodate for the different media requirements of muscle and neuron cells.

The applications of 3D printing technology coupled with micro-fluidics to generate $3 \mathrm{D}$ in vitro muscle models, as demonstrated by previously mentioned examples, possess significant potential for the future of muscle development studies, disease modeling, and drug screening compared to 2D culture models. However, the ability to create a reproducible, high throughput in vitro muscle model for disease modeling and drug screening remains a huge challenge. 


\section{D printed in vitro model in toxicology: challenge and perspectives}

With the development of both 3D culture and bio-fabrication technologies, 3D in vitro tissue models have been recognized as an advanced approach for toxicity studies as they provide controllable variations to identify mechanisms of treatment response; close imitation of the microenvironment and physiology of the native tissue; and high efficiency and throughput of model establishment. In an early study, HepaRG and HepG2 spheroids (Fig. 3A, B) were fabricated with a hanging drop system and tested for toxicity response against aflatoxin B1, amiodarone, valproic and chlorpromazine. Compared to the $2 \mathrm{D}$ culture, the spheroids showed increased metabolic activities as well as increased gluconeogenesis, cell polarization, and diffusion barrier effect of ECM with the drugs of interest. These distinctions in the drug response between 2D and 3D models demonstrate the importance of the in vivo environment in evaluating toxicity (Mueller et al. 2014). Parallelly, Ramaiahgari et al. (2014) used Matrigel to 3D culture HepG2 cells. The size of the spheroids was regulated by the bottom surface area of the well plate and the amounts of cells. Increased cell polarity, functionality and thereby the sensitivity to the hepatotoxic drugs were also observed.
Fig. 3 Evolution of in vitro model of complex tissue. A, B Early liver spheroid culture (Mueller et al. 2014); C, D liver spheroid culture for in situ quantification (Hong and Song 2021); E-H 3D printed vascularized liver model (Massa et al. 2017)
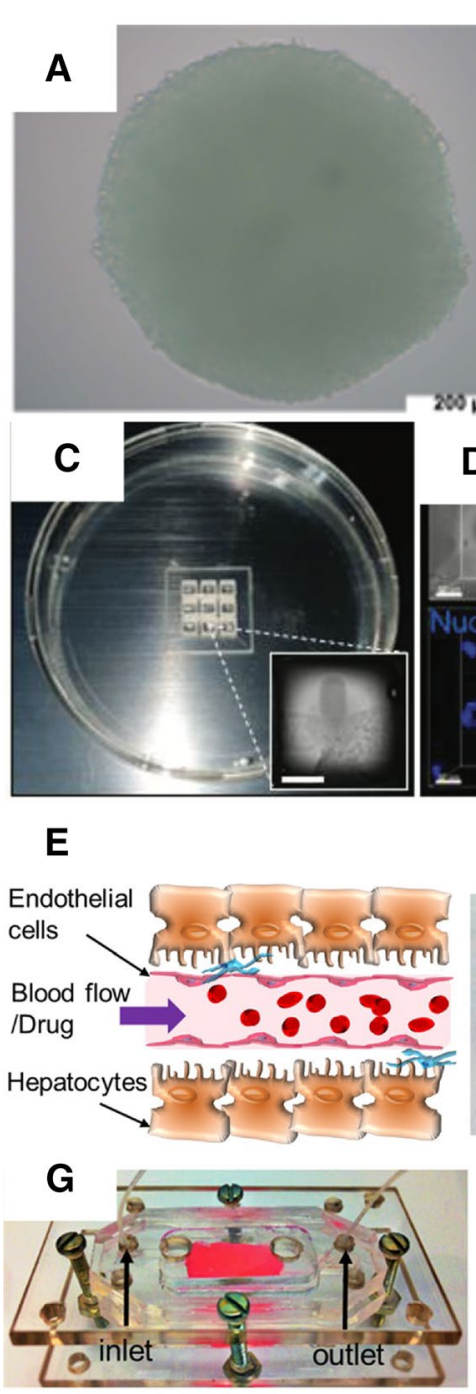

Top view

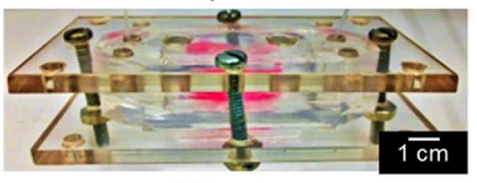

Side view
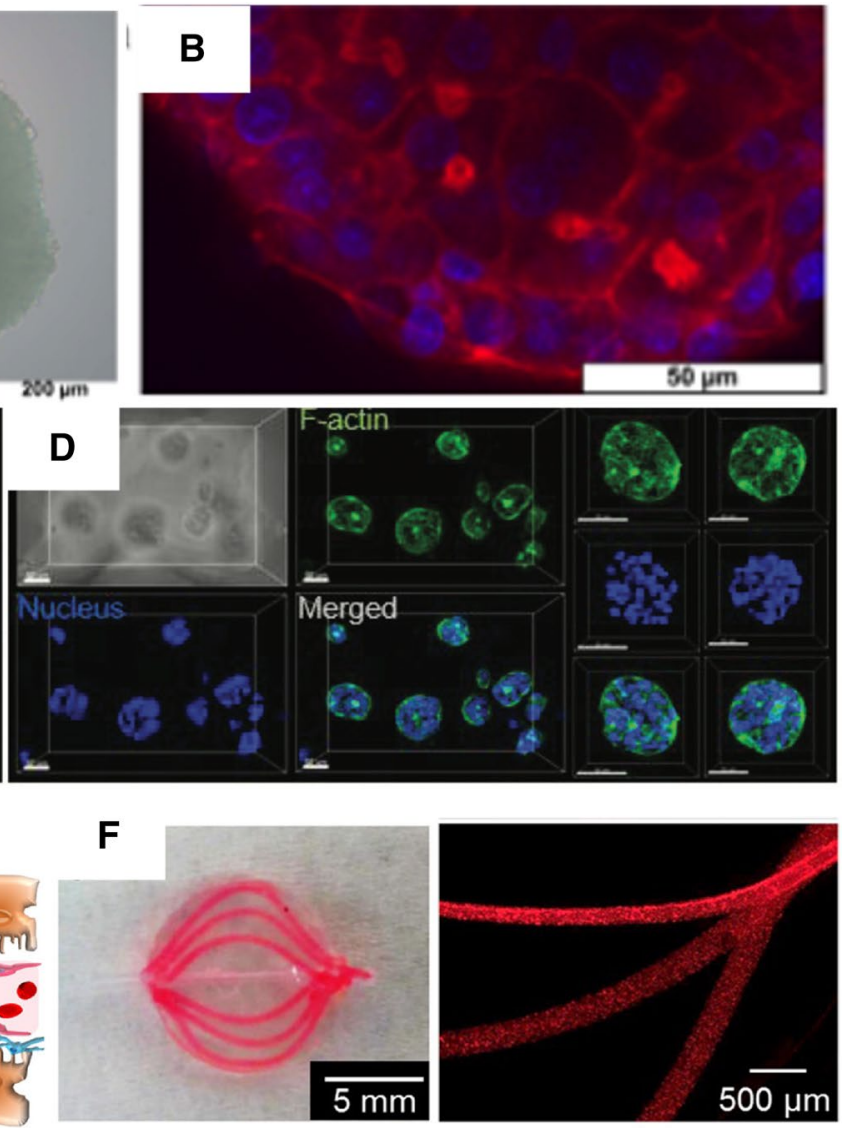

$\mathbf{H}$

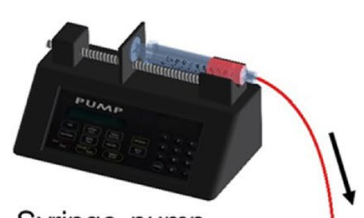

Syringe pump 
For better control over the remodeling and architecture recapture of the complex tissue, 3D printing has been involved in the production of the in vitro models, resulting in an improved mimicry of the biochemical, histological and functional recapitulation, as well as addressing the different study purposes. For example, micro-extrusion-based printing was employed to optimize a $3 \mathrm{D}$ liver in vitro model. Alginate, gelatin and Matrigel were blended at an optimized ratio for printability, cell viability, metabolism and long-term stability of the scaffold. The viability and secretion of albumin were maintained up to 21 days, indicating the potential of the model for chronic toxicity testing (Schmidt et al. 2020). On the other hand, the rapid yet well-controlled production of the liver spheroid by $3 \mathrm{D}$ printing enabled the quantitative evaluation of drug-induced toxicity in an in situ manner (Fig. 3C, D), which can play an accelerating role in the long and expensive drug development process (Hong and Song 2021). One of the important complexed features of the native tissues, which should be taken into consideration for toxicity assessment, is that they are highly vascularized (Fig. 3E). Utilizing extrusion-based 3D printing, Massa et al. fabricated 3D vascularized liver model with perfusable channels (Fig. 3F-H) involving an endothelial layer that barriers the diffusion of molecules. The model was tested for the toxicity study of acetaminophen, which has been revealed to damage the endothelial cells in the liver sinusoid. In this model, the toxicity effect of the drug was tested on the endothelial layer and the HepG2/C3A cell protected by the endothelial layer, better mimicking the in vivo exposure scenario (Massa et al. 2017).

While 3D bio-printing offers many advantages, there are still challenges and limitations to overcome, as well as room to improve.

To successfully replicate complicated tissue in vitro, a model should not only reproduce its histological and biochemical characteristics, but also its functions, such as metabolite exchange, nutrient transportation and contraction. Ahn et al., for example, constructed a 3D heart cantilever based on rat cardiomyocytes, in which a piece of cardiac tissue was remodeled, and the contraction function was recapitulated and evaluated. Dosed with titanium dioxide at 100 $\mu \mathrm{g} / \mathrm{mL}$, they observed a gradual decrease in the contractile force, and the spontaneous beating ceased in $24 \mathrm{~h}$ (Ahn et al. 2018). The result did not align with a previous study with a $2 \mathrm{D}$ culture model, where the spontaneous beating of the rat cardiomyocytes was maintained despite the reduced beating rate and amplitude (Jawad et al. 2011). There are a few hypotheses as to why the 3D heart tissue stopped beating, including: (1) cardiomyocytes are more resistant to the titanium dioxide toxicity in 2D than in 3D culture; (2) the remaining contractility of the cardiomyocytes after dosing was enough to be observed on the single cell level, but not enough to support the beating function; and (3) the toxicity toward the tissue ECM was reflected in the 3D model but neglected in 2D culture. In any case, the difference between the two studies emphasizes the importance of function recapitulation in the in vitro modeling. However, the gap between the exquisite native tissue and our limited understanding of the in vivo conditions and appropriate fabrication technology remains a key difficulty and hot topic in ongoing research.

\section{Tissue complexity}

The complexity of the tissue is one of the challenges in reproducing a native tissue. Regardless of the biomaterial sources available, it remains a challenge to fully represent the biophysical and biochemical features of the native ECM in an artificial bio-microenvironment. One solution for the dilemma is to incorporate AECM in the biomaterial applied in the 3D printing. As reported by Ma et al. (2018a, b), the photo-patterning of cells and stiffness of the scaffold were precisely regulated via $3 \mathrm{D}$ printing of the $\mathrm{dECM}$-blended bioink to provide biomimetic physical signals to the cells, while the biochemical cues embedded in the native liver ECM were simultaneously delivered. Similar results were achieved in a later study by another group (Mao et al. 2020). For control of the biochemical cues individually in the scaffold, highly engineered synthetic material customized with biomolecules of interest has been developed to facilitate the establishment of 3D culture systems. In an early collective study conducted by Taubenberger et al., PEG was decorated with biochemical mediators, such as metalloproteinasecleavage spot, ECM mimicking cell adhesion peptides and a set of growth factors. The highly programmable platform they developed was able to create an in vitro bio-microenvironment with multiplexed biochemical cues and controlled mechanical properties of the matrix (Taubenberger et al. 2016). Another challenge is that the ECM is highly dynamic. Material properties must be carefully tuned to match the progression of the in vitro model and physiological process. For example, in the cardiac micro-tissue fabricated via DLP printing, the crosslinking density of the scaffold made of GelMA was carefully tuned, so that the degradation rate of the scaffold matched the ECM deposition of human cardiac fibroblasts (HCF), which essentially supported the maturation and contraction phenotype of the artificial tissue over 7 days (Miller et al. 2021). To improve the mimicry, a dynamic culture system could be combined with the artificial tissue. For example, a microfluidic chip with pressure channels was designed for colon tumor organoid culture to mimic the peristalsis, which is an important feature of their native microenvironment. The proliferation and organoid size were significantly increased compared to static culture as the media flowed through the pressure channels and mechanical 
stimulus was delivered. The peristalsis-stimulated organoid also showed decreased uptake and response to ellipticineladen micelle dosing, implying that genuine recapitulation of ECM dynamics may have a significant impact on the in vitro model's drug/toxin response (Fang et al. 2021).

On the other hand, native tissue has a complex cell composition, whereas much of the earlier research used only a few cell lines. It is an effective approach to simplify the model for certain scientific problems, but it hinders the recapitulation of tissue integrity, functionality, and response to drug/toxin doses. Organoid and in situ differentiation offer significant advantages in breaking down this barrier. $\mathrm{Gu}$ et al. created 3D neuron constructs from human neuron stem cells using extrusion-based 3D printing. After in situ differentiation of the stem cells, a neuron micro-tissue composed of functional neurons and supporting neuroglia was obtained ( $\mathrm{Gu}$ et al. 2016). A follow-up study using iPSCs was conducted by the same group, demonstrating the strategy's potential for application in a variety of tissues $(\mathrm{Gu}$ et al. 2017). This method is particularly useful for models that require difficult-to-obtain cell sources. For example, because of the non-proliferating nature of cardiomyocytes and the requirement for high cardiomyocyte density, it has been difficult to establish an in vitro cardiac model in a large dimension and with integrated anatomy structures. Kupfer et al. (2020) used in situ differentiation of 3D printing iPSCs to create a cardiac organoid with highly biomimetic chamber structure and pumping activities on a $1.3 \mathrm{~cm}$ scale. While the organoid technology and in situ differentiation provide a versatile strategy to achieve multicellular construct, it is difficult to control the ratio of the cellular component. For designated cell composition, 3D printing could also be exploited as it provides high resolution for cell deposition. Focusing on the subject has resulted in significant progress. Because of their high yet biocompatible printing resolution, light-assisted printing processes, such as stereolithography and DLP printing, are intrinsically advantageous for multicellular printing. Furthermore, multiplexed printing systems have been developed to aid the facile multicellular printing; for example, multi-head and core-shell structured nozzles for extrusion and inkjet-based bioprinting have been developed. In place of the distinct flow mechanics at the microscale, microfluidic-based strategies can also be incorporated to the conventional 3D printing processes for precise control on the heterogeneity of the bioink. Ashammakhi et al. (2019) have provided a more in-depth review on the topic.

\section{Systematic recapitulation}

Another gap to be filled is the lack of mimicry on the systematic level. Many pharmacological and toxicological studies are hampered by poor in vitro-in vivo correlation because the response is usually generated by organ-organ interaction rather than a single organ or tissue. To address this concern, multi-organ-on-a-chip models incorporating multiple tissue models linked to a shared medium circulation system have been developed. Microfluidic devices have emerged as a powerful platform with individual chambers for the culture of each tissue and controlled flow of media enabled by designated channels, and extensive work has been devoted to such culture systems, resulting in improved drug response prediction outcomes (Sung 2021). Further improvement could be achieved by incorporating the evolving in vitro tissue models as discussed above as well as more customized and tailored design in the microfluidic devices for better mimicry of the flow dynamics and substance exchange (Goldstein et al. 2021).

In addition, it is necessary for systematic modeling to involve the in vitro recapitulation of the immune system, which plays a critical role in many disease progression and pharmacological/toxicological responses, and has become increasingly important in the development of therapeutics for many conditions. Keeping this in mind, recent studies have focused on incorporating immune components into in vitro models and have achieved effective results. iPSC and THP-1-derived macrophages, for example, were incorporated into the in vitro glioblastoma microenvironment model to mimic the infiltration of macrophage alongside microglia via DLP printing. The engagement of the macrophages resulted in a significantly more authentic recapitulation of the original tissue, which had a substantial impact on the drug response of the model (Tang et al. 2020). THP-1 was also used to investigate the general pro-inflammatory response in a human-based multi-organ-on-a-chip model which involved hepatocyte, cardiomyocyte and skeletal muscle myoblasts (Sasserath et al. 2020). However, most of the work has been devoted to the innate immune system, while the in vitro modeling of the adaptive immune system remains to be explored. In fact, the state-of-the-art in vitro modeling of the immune system is still in its early stages. A more in-depth and comprehensive review regarding the topic has been provided by Polini et al. (2019).

\section{Microarchitecture}

Furthermore, due to the limited resolution of bioprinting and cell deposition, it is difficult to recreate the microarchitecture and hierarchical nature of the tissue, which is particularly important in the integrated functionality of certain organs, such as the lungs, kidneys, and livers. The alveoli in the lungs, for example, are physically air sacs held and entangled by a network of capillaries (Fig. 4A). This extremely sophisticated structure is necessary for the exchange of oxygen and carbon dioxide between the pulmonary and circulatory systems, but it also adds a significant challenge to the fabrication process, particularly with soft materials. 
Fig. 4 A hierarchical and entangled structure of human lung alveolus (Huh et al. 2012); B the air-liquid interface model based on a PDMS chamber (Huh et al. 2012); C genuine recapitulation of the complexed alveolus microstructure by photo-absorbent-assisted stereolithography (Grigoryan et al. 2019)
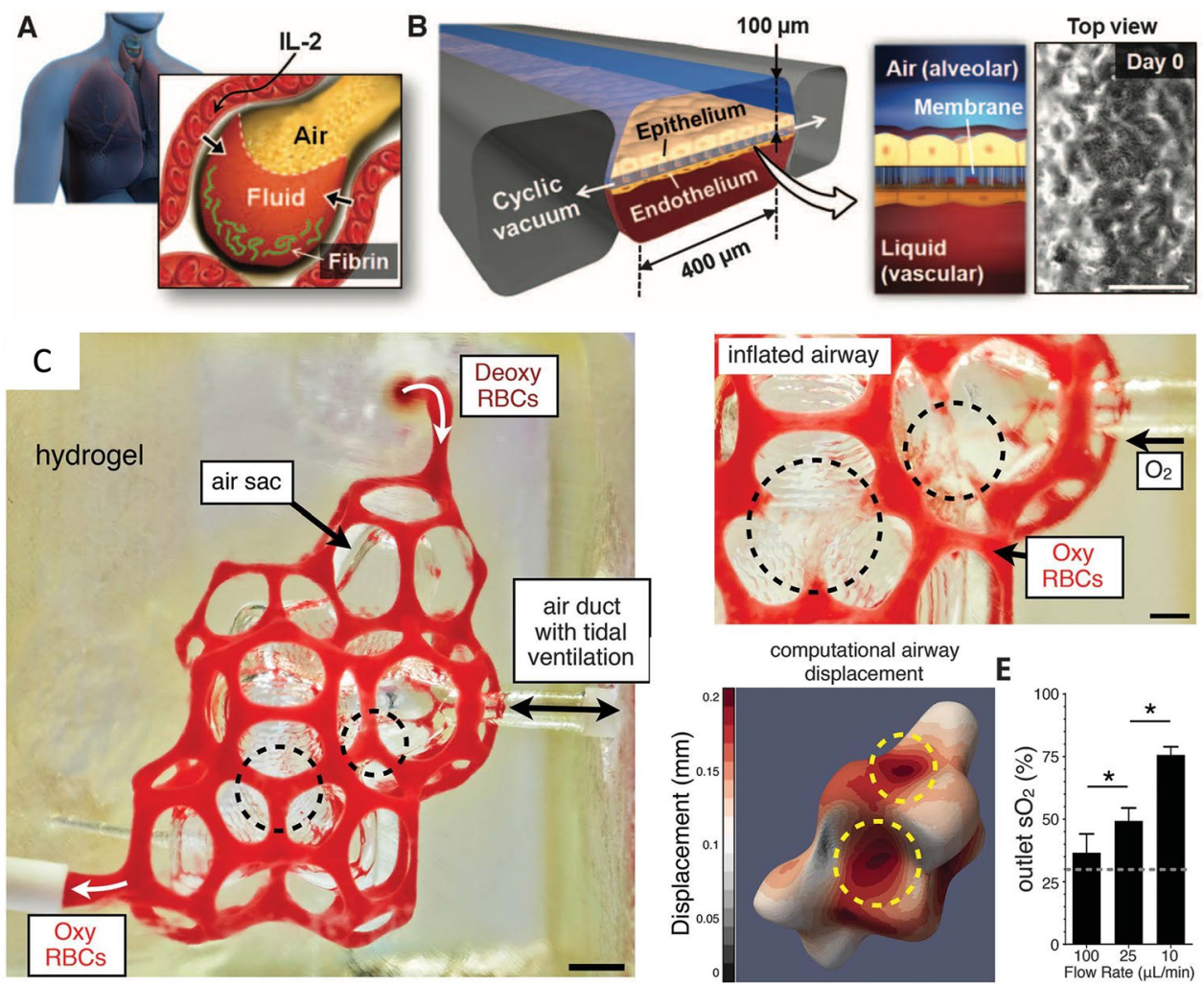

computational airway
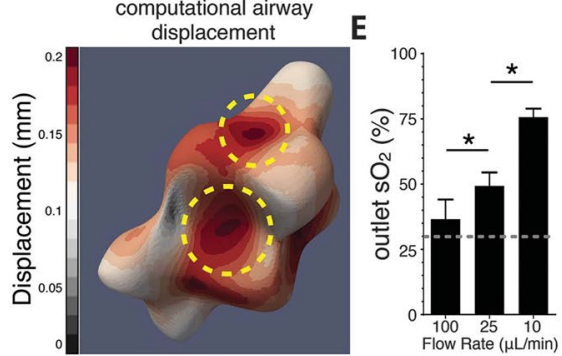

Earlier studies focused on multilayer 3D culture integrated on a PDMS chamber to capture the air-liquid interface in the alveolus for disease modeling and drug testing (Fig. 4B) (Benam et al. 2016; Huh et al. 2012). While the biochemical and histological characteristics of the tissue were successfully replicated to some extent, the membrane thickness of the blood vessels and the cell-liquid ratio were not fully recapitulated, resulting in inaccurate capture of tissue metabolism and biochemical signaling (Shrestha et al. 2020). The advanced $3 \mathrm{D}$ printing technique can improve the recapitulation of the alveolar microarchitecture. Innovatively applying food dye as a photo-absorber, Grigoryan et al. enhanced the printing resolution of soft hydrogel, PEGDA $\left(M_{\mathrm{w}}=6 \mathrm{kDa}\right)$, in DLP-based 3D printing (Fig. 4C). This allowed the printing of the complexed microstructure of alveolar with a biocompatible bioink, in which oxygenation and flow of human red blood cells was supported and vascularization of human umbilical vein endothelial cells (HUVECs) was achieved (Grigoryan et al. 2019). One limitation of this study is that the native alveolar cell composition was not included or tested, which limits the model's application in drug and toxin studies. Nonetheless, it is stated that the recapitulation of the exquisite native tissue's microarchitecture is within reach owing to the rising resolution of $3 \mathrm{D}$ printing techniques and a wider range of biomaterials.

\section{Vascularization}

As the nature route of nutrient and oxygen transportation and an essential part in the induction of organogenesis (Ding et al. 2010), vasculatures remain a stumbling block in both the maturation of organoids (Vargas-Valderrama et al. 2020) and the 3D fabrication of larger in vitro tissues. Currently, the major approach to introduce the vascularization to an in vitro model is to involve a sacrificial template. For example, Skylar-Scott et al. proposed a sacrificial writing into functional tissue (SWIFT) method, in which they compact a large volume of organoids in a thermal-gelling ECM and directly write the vascular channels with gelatin ink in an embedding manner at $0-4{ }^{\circ} \mathrm{C}$. After printing, the gelatin was removed by perfusion as the construction was warmed up to $37^{\circ} \mathrm{C}$ for future culture. This novel strategy enabled addition of vasculature and large-dimension culture of organoids (Skylar-Scott et al. 2019). The sacrificial gelatin ink could also facilitate direct printing of vasculatures in a coaxial extrusion-based 3D printing set-up. As reported by Shao et al. perfusable core-shell constructs with a scale larger than $1 \mathrm{~cm}$ have been printed with HUVECs mixed in the inner gelatin phase and tissue cells (human breast cancer cell MDA-MB-231 and mouse osteoblast MC3T3-E1) laden in the outer GelMA phase. After removal of gelatin and auto-seeding of HUVECs, the construct remodeled into a vasculature and was cultured for over 20 days (Shao et al. 
2020). The phase-transition removal of gelatin is more biocompatible to avoid unwanted chemical stimulation compared to alginate-Ca and Pluronic, which are commonly used in earlier work, but the temperature shock involved in this process should be taken into consideration for specific cells and models (Lindquist and Mertens 2018). Moreover, the process to remove the template still hinders the efficient fabrication of the in vitro model. In contrast, DLP printing remains advantageous to fabricate vasculatures directly and rapidly. Zhu et al. reported the DLP-based continuous bioprinting of a pre-vascularized in vitro tissue with gradient channel diameter and biomimetic branching configuration. Facilitated with a motorized syringe pump, each construct was printed within $1 \mathrm{~min}$; lumen-like structure and tight junctions of the endothelium was developed after culture for 1 week (Zhu et al. 2017). Despite the evolving techniques to produce vascularized perfusable channels, most work was conducted with primary HUVECs, which are isolated from an identical organ and stage of human life (Cao et al. 2017). However, the endothelium is highly heterogeneous throughout the different organs in terms of histological features, metabolism, angiogenesis and involvement in the immune response (Przysinda et al. 2020). It is noteworthy that in the development of an organ-specific vasculature, the diverse phenotypes of the endothelium should be recognized and well characterized.

\section{High throughput}

High-throughput screening (HTS) systems are widely used by the pharmaceutical industry as an efficient method to process large numbers of compounds and molecular targets in a rapid manner. As such, pharmaceutical companies are turning to the use of 3D biomimetic human tissues in HTS format for preclinical toxicity testing of potential drug candidates. 3D bioprinting approaches, such as inkjet and micro-extrusion-based processes, are limited in scalability, resolution, patterning flexibility, and/or speed, which makes them unsuitable for high-throughput fabrication of complex cell-laden 3D microstructures within multi-well plates commonly used in HTS systems for drug screening and assay development. These shortcomings severely limit the widespread adoption of 3D printed cell culture platforms since researchers often rely on products configured to interface with commonly used lab instruments and equipment. Recently, DLP printing has made HTS possible. With rational integration of the micro-continuous projection printing and the automated well plate registration, Hwang et al. managed to fabricate complex 3D bio-constructs directly in a well plate rapidly and consistently. Depending on the complexity of the tissue construct, the total fabrication time for a fully populated 96-well plate typically ranges from 20 to 40 min (Hwang et al. 2021). Future work for printing in a 384-well plate or more wells is yet to be developed for HTS applications.

\section{Concluding remarks}

3D bioprinting has made significant progress in in vitro recapitulation of complex tissues. By applying an appropriate printing technique and biomaterial, accurate recapture of the ECM composition, cell population, bio-microenvironment, organ microarchitecture, and tissue functionality has been achieved in a variety of tissues, such as cancer, heart, muscle, liver, kidney, and will continue to be broadened and enhanced as the studies go on. Despite the encouraging results being achieved, it is noteworthy that they are usually labor and technique intensive. To bridge the gap between fundamental research and pragmatic applications, the scalability and reproducibility of the modeling strategies must also be addressed. In addition, with the arising need in the evaluation of therapeutic and substance in subdivided population and in the point-of-care scenario, the required time of production of personalized in vitro model must also be improved.

Acknowledgements This work was supported in part by grants from the National Institutes of Health (R01CA253615, R21AR074763, R21HD100132, R33HD090662) and National Science Foundation (1903933, 1937653). The authors would like to thank Mr. Henry Hwang, Mr. Jacob Schimelman, Dr. David Berry and Ms. Emmie Yao for their help on editing and proofreading.

\section{Declarations}

Conflict of interest SC is a co-founder of and has an equity interest in Allegro 3D, Inc., and he serves on the scientific advisory board. Some of his research grants, including those acknowledged here, have been identified for conflict of interest management based on the overall scope of the project and its potential benefit to Allegro 3D, Inc. The author is required to disclose this relationship in publications acknowledging the grant support; however, the research subject and findings reported here did not involve the company in any way and have no relationship with the business activities or scientific interests of the company. The terms of this arrangement have been reviewed and approved by the University of California San Diego in accordance with its conflict of interest policies. The other authors have no competing interests to declare.

Open Access This article is licensed under a Creative Commons Attribution 4.0 International License, which permits use, sharing, adaptation, distribution and reproduction in any medium or format, as long as you give appropriate credit to the original author(s) and the source, provide a link to the Creative Commons licence, and indicate if changes were made. The images or other third party material in this article are included in the article's Creative Commons licence, unless indicated otherwise in a credit line to the material. If material is not included in the article's Creative Commons licence and your intended use is not permitted by statutory regulation or exceeds the permitted use, you will need to obtain permission directly from the copyright holder. To view a copy of this licence, visit http://creativecommons.org/licenses/by/4.0/. 


\section{References}

Ahn S, Ardoña HAM, Lind JU, Eweje F, Kim SL, Gonzalez GM, Liu Q, Zimmerman JF, Pyrgiotakis G, Zhang Z, Beltran-Huarac J, Carpinone P, Moudgil BM, Demokritou P, Parker KK (2018) Mussel-inspired 3D fiber scaffolds for heart-on-a-chip toxicity studies of engineered nanomaterials. Anal Bioanal Chem 410(24):61416154. https://doi.org/10.1007/s00216-018-1106-7[CS11]

Ashammakhi N, Ahadian S, Xu C, Montazerian H, Ko H, Nasiri R, Barros N, Khademhosseini A (2019) Bioinks and bioprinting technologies to make heterogeneous and biomimetic tissue constructs. Materials Today Bio. https://doi.org/10.1016/j.mtbio. 2019.100008

Benam KH, Villenave R, Lucchesi C, Varone A, Hubeau C, Lee HH, Alves SE, Salmon M, Ferrante TC, Weaver JC, Bahinski A, Hamilton GA, Ingber DE (2016) Small airway-on-a-chip enables analysis of human lung inflammation and drug responses in vitro. Nat Methods 13(2):151-157. https://doi.org/10.1038/nmeth.3697

Brack A, Bakooshli MA, Lippmann ES, Mulcahy B, Iyer N, Nguyen CT, Tung K, Stewart BA, van den Dorpel H, Fuehrmann T, Shoichet M, Bigot A, Pegoraro E, Ahn H, Ginsberg H, Zhen M, Ashton RS, Gilbert PM (2019) A 3D culture model of innervated human skeletal muscle enables studies of the adult neuromuscular junction. Elife 8:e44530. https://doi.org/10.7554/eLife. 44530.001

Braun NJ, Galaska RM, Jewett ME, Krupa KA (2021) Implementation of a dynamic co-culture model abated silver nanoparticle interactions and nanotoxicological outcomes in vitro. Nanomaterials. https://doi.org/10.3390/nano11071807

Cammarata CR, Hughes ME, Ofner CM (2015) Carbodiimide induced cross-linking, ligand addition, and degradation in gelatin. Mol Pharm 12(3):783-793. https://doi.org/10.1021/mp5006118

Cao Y, Gong Y, Liu L, Zhou Y, Fang X, Zhang C, Li Y, Li J (2017) The use of human umbilical vein endothelial cells (HUVECs) as an in vitro model to assess the toxicity of nanoparticles to endothelium: a review. J Appl Toxicol 37(12):1359-1369. https:// doi.org/10.1002/jat.3470

Cao H, Cheng HS, Wang JK, Tan NS, Tay CY (2021) A 3D physiomimetic interpenetrating network-based platform to decode the pro and anti-tumorigenic properties of cancer-associated fibroblasts. Acta Biomater 132:448-460. https://doi.org/10.1016/j. actbio.2021.03.037

Cappello V, Francolini M (2017) Neuromuscular junction dismantling in amyotrophic lateral sclerosis. Int J Mol Sci. https://doi.org/10. 3390/ijms 18102092

Chen H, Cheng Y, Wang X, Wang J, Shi X, Li X, Tan W, Tan Z (2020) $3 \mathrm{D}$ printed in vitro tumor tissue model of colorectal cancer. Theranostics 10(26):12127-12143. https://doi.org/10.7150/ thno. 52450

Claeyssens F, Hasan EA, Gaidukeviciute A, Achilleos DS, Ranella A, Reinhardt C, Ovsianikov A, Shizhou X, Fotakis C, Vamvakaki M, Chichkov BN, Farsari M (2009) Three-dimensional biodegradable structures fabricated by two-photon polymerization. Langmuir 25(5):3219-3223. https://doi.org/10.1021/la803803m

Davila JC, Rodriguez RJ, Melchert RB, Acosta D (1998) Predictive value of in vitro model systems in toxicology. Annu Rev Pharmacol Toxicol 38:63-96. https://doi.org/10.1146/annurev.pharm tox.38.1.63

Derakhshanfar S, Mbeleck R, Xu K, Zhang X, Zhong W, Xing M (2018) 3D bioprinting for biomedical devices and tissue engineering: a review of recent trends and advances. Bioact Mater 3(2):144-156. https://doi.org/10.1016/j.bioactmat.2017.11.008

Ding B, Nolan DJ, Butler JM, James D, Babazadeh AO, Rosenwaks Z, Mittal V, Kobayashi H, Shido K, Lyden D, Sato TN, Rabbany
SY, Rafii S (2010) Inductive angiocrine signals from sinusoidal endothelium are required for liver regeneration. Nature 468(7321):310-315. https://doi.org/10.1038/nature09493

Duchamp M, Liu T, van Genderen AM, Kappings V, Oklu R, Ellisen LW, Zhang YS (2019) Sacrificial bioprinting of a mammary ductal carcinoma model. Biotechnol J. https://doi.org/10.1002/ biot. 201700703

Duval K, Grover H, Han LH, Mou Y, Pegoraro AF, Fredberg J, Chen $\mathrm{Z}$ (2017) Modeling physiological events in 2D vs. 3D cell culture. Physiology 32(4):266-277. https://doi.org/10.1152/physi ol.00036.2016

Dvir T, Timko BP, Brigham MD, Naik SR, Karajanagi SS, Levy O, Jin H, Parker KK, Langer R, Kohane DS (2011) Nanowired threedimensional cardiac patches. Nat Nanotechnol 6(11):720-725. https://doi.org/10.1038/nnano.2011.160

Fang G, Lu H, Al-Nakashli R, Chapman R, Zhang Y, Ju LA, Lin G, Stenzel MH, Jin D (2021) Enabling peristalsis of human colon tumor organoids on microfluidic chips. Biofabrication. https:// doi.org/10.1088/1758-5090/ac2ef9

Faustino Martins JM, Fischer C, Urzi A, Vidal R, Kunz S, Ruffault PL, Kabuss L, Hube I, Gazzerro E, Birchmeier C, Spuler S, Sauer S, Gouti M (2020) Self-organizing 3D human trunk neuromuscular organoids. Cell Stem Cell 26(2):172-186.e6. https://doi.org/10. 1016/j.stem.2019.12.007

Ferri N, Siegl P, Corsini A, Herrmann J, Lerman A, Benghozi R (2013) Drug attrition during pre-clinical and clinical development: understanding and managing drug-induced cardiotoxicity. Pharmacol Ther 138(3):470-484. https://doi.org/10.1016/j. pharmthera.2013.03.005

Fleszar AJ, Walker A, Porubsky V, Flanigan W, James D, Campagnola PJ, Weisman PS, Kreeger PK (2018) The extracellular matrix of ovarian cortical inclusion cysts modulates invasion of fallopian tube epithelial cells. APL Bioeng. https://doi.org/10.1063/1. 5022595

García-Astrain C, Gandini A, Peña C, Algar I, Eceiza A, Corcuera M, Gabilondo N (2014) Diels-Alder "click" chemistry for the crosslinking of furfuryl-gelatin-polyetheramine hydrogels. RSC Adv 4(67):35578-35587. https://doi.org/10.1039/c4ra06122e

Gasiorowski JZ, Murphy CJ, Nealey PF (2013) Biophysical cues and cell behavior: the big impact of little things. Annu Rev Biomed Eng 15:155-176. https://doi.org/10.1146/annur ev-bioeng-071811-150021

Goldstein Y, Spitz S, Turjeman K, Selinger F, Barenholz Y, Ertl P, Benny O, Bavli D (2021) Breaking the third wall: Implementing 3d-printing technics to expand the complexity and abilities of multi-organ-on-a-chip devices. Micromachines. https://doi.org/ 10.3390/mi12060627

Gori M, Giannitelli SM, Torre M, Mozetic P, Abbruzzese F, Trombetta M, Traversa E, Moroni L, Rainer A (2020) Biofabrication of hepatic constructs by $3 \mathrm{~d}$ bioprinting of a cell-laden thermogel: an effective tool to assess drug-induced hepatotoxic response. Adv Healthc Mater. https://doi.org/10.1002/adhm.202001163

Goulart E, de Caires-Junior LC, Telles-Silva KA, Araujo BHS, Rocco SA, Sforca M, de Sousa IL, Kobayashi GS, Musso CM, Assoni AF, Oliveira D, Caldini E, Raia S, Lelkes PI, Zatz M (2020) $3 \mathrm{D}$ bioprinting of liver spheroids derived from human induced pluripotent stem cells sustain liver function and viability in vitro. Biofabrication. https://doi.org/10.1088/1758-5090/ab4a30

Grefte S, Kuijpers-Jagtman AM, Torensma R, von Den Hoff JW (2007) Skeletal muscle development and regeneration. Stem Cells Dev 16(5):857-868. https://doi.org/10.1089/scd.2007.0058

Greising SM, Corona BT, McGann C, Frankum JK, Warren GL (2019) Therapeutic approaches for volumetric muscle loss injury: a systematic review and meta-analysis. Tissue Eng Part B Rev 25(6):510-525. https://doi.org/10.1089/ten.teb.2019.0207 
Grigoryan B, Paulsen SJ, Corbett DC, Sazer DW, Fortin CL, Zaita AJ, Greenfield PT, Calafat NJ, Gounley JP, Ta AH, Johansson F, Randles A, Rosenkrantz JE, Louis-Rosenberg JD, Galie PA, Stevens KR, Miller JS (2019) Multivascular networks and functional intravascular topologies within biocompatible hydrogels. Science 364(6439):458-464. https://doi.org/10.1126/science.aav9750

Grix T, Ruppelt A, Thomas A, Amler AK, Noichl BP, Lauster R, Kloke L (2018) Bioprinting perfusion-enabled liver equivalents for advanced organ-on-a-chip applications. Genes. https://doi.org/ 10.3390/genes 9040176

Grosberg A, Alford PW, McCain ML, Parker KK (2011) Ensembles of engineered cardiac tissues for physiological and pharmacological study: Heart on a chip. Lab Chip 11(24):4165-4173. https://doi. org/10.1039/c1lc20557a

Gu Q, Tomaskovic-Crook E, Lozano R, Chen Y, Kapsa RM, Zhou Q, Wallace GG, Crook JM (2016) Functional 3D neural mini-tissues from printed gel-based bioink and human neural stem cells. Adv Healthc Mater 5(12):1429-1438. https://doi.org/10.1002/adhm. 201600095

Gu Q, Tomaskovic-Crook E, Wallace GG, Crook JM (2017) 3D bioprinting human induced pluripotent stem cell constructs for in situ cell proliferation and successive multilineage differentiation. Adv Healthc Mater. https://doi.org/10.1002/adhm.20170 0175

Gudapati H, Dey M, Ozbolat I (2016) A comprehensive review on droplet-based bioprinting: past, present and future. Biomaterials 102:20-42. https://doi.org/10.1016/j.biomaterials.2016. 06.012

Guo Y, Pu WT (2020) Cardiomyocyte maturation: new phase in development. Circ Res. https://doi.org/10.1161/CIRCRESAHA.119. 315862

Guo X, Smith V, Jackson M, Tran M, Thomas M, Patel A, Lorusso E, Nimbalkar S, Cai Y, McAleer CW, Wang Y, Long CJ, Hickman JJ (2020) A human-based functional NMJ system for personalized ALS modeling and drug testing. Adv Ther 3(11):2000133. https://doi.org/10.1002/adtp.202000133

Hakobyan D, Médina C, Dusserre N, Stachowicz ML, Handschin C, Fricain JC, Guillermet-Guibert J, Oliveira H (2020) Erratum: Laser-assisted 3D bioprinting of exocrine pancreas spheroid models for cancer initiation study (Biofabrication (2020) 12 (035001) DOI: 10.1088/1758-5090/ab7cb8). Biofabrication. https://doi.org/10.1088/1758-5090/aba1fb

Heart Disease and Stroke Statistics (2021) Update: a report from the American Heart Association. Circulation. https://doi.org/10. 1161/CIR.0000000000000950

Hermida MA, Kumar JD, Schwarz D, Laverty KG, di Bartolo A, Ardron M, Bogomolnijs M, Clavreul A, Brennan PM, Wiegand UK, Melchels FP, Shu W, Leslie NR (2020) Three dimensional in vitro models of cancer: Bioprinting multilineage glioblastoma models. Adv Biol Regul. https://doi.org/10.1016/j.jbior.2019. 100658

Hiller T, Berg J, Elomaa L, Röhrs V, Ullah I, Schaar K, Dietrich AC, Al-Zeer MA, Kurtz A, Hocke AC, Hippenstiel S, Fechner H, Weinhart M, Kurreck J (2018) Generation of a 3D liver model comprising human extracellular matrix in an alginate/gelatinbased bioink by extrusion bioprinting for infection and transduction studies. Int J Mol Sci. https://doi.org/10.3390/ijms19103129

Hinson JT, Chopra A, Nafissi N, Polacheck WJ, Benson CC, Swist S, Gorham J, Yang L, Schafer S, Sheng CC, Haghighi A, Homsy J, Hubner N, Church G, Cook SA, Linke WA, Chen CS, Seidman JG, Seidman CE (2015) Titin mutations in iPS cells define sarcomere insufficiency as a cause of dilated cardiomyopathy. Science 349(6251):982-986. https://doi.org/10.1126/science. aaa5458
Hirt MN, Hansen A, Eschenhagen T (2014) Cardiac tissue engineering: state of the art. Circ Res 114(2):354-367. https://doi.org/10. 1161/CIRCRESAHA.114.300522

Hong S, Song JM (2021) A 3D cell printing-fabricated HepG2 liver spheroid model for high-content in situ quantification of druginduced liver toxicity. Biomater Sci 9(17):5939-5950. https:// doi.org/10.1039/d1bm00749a

Hopp B (2012) Femtosecond laser printing of living cells using absorbing film-assisted laser-induced forward transfer. Opt Eng 51(1):014302. https://doi.org/10.1117/1.oe.51.1.014302

Hribar KC, Finlay D, Ma X, Qu X, Ondeck MG, Chung PH, Zanella F, Engler AJ, Sheikh F, Vuori K, Chen SC (2015) Nonlinear 3D projection printing of concave hydrogel microstructures for longterm multicellular spheroid and embryoid body culture. Lab Chip 15(11):2412-2418. https://doi.org/10.1039/c5lc00159e

Huh D, Leslie DC, Matthews BD, Fraser JP, Jurek S, Hamilton GA, Thorneloe KS, Mcalexander MA, Ingber DE (2012) A human disease model of drug toxicity-induced pulmonary edema in a lung-on-a-chip microdevice. Sci Transl Med 10(449):eaau4555. https://doi.org/10.1126/scitranslmed.aau4555

Hwang HH, You S, Ma X, Kwe L, Victorine G, Lawrence N, Wan X, Shen H, Zhu W, Chen S (2021) High throughput direct 3D bioprinting in multiwell plates. Biofabrication. https://doi.org/ 10.1088/1758-5090/ab89ca

Jawad H, Boccaccini AR, Ali NN, Harding SE (2011) Assessment of cellular toxicity of $\mathrm{TiO} 2$ nanoparticles for cardiac tissue engineering applications. Nanotoxicology 5(3):372-380. https://doi. org/10.3109/17435390.2010.516844

Kang D, Hong G, An S, Jang I, Yun WS, Shim JH, Jin S (2020) Bioprinting of multiscaled hepatic lobules within a highly vascularized construct. Small. https://doi.org/10.1002/smll.201905505

Kelly BE, Bhattacharya I, Heidari H, Shusteff M, Spadaccini CM, Taylor HK (2019) Volumetric additive manufacturing via tomographic reconstruction. Science 363(6431):1075-1079. https:// doi.org/10.1126/science.aau7114

Kim MK, Jeong W, Lee SM, Kim JB, Jin S, Kang HW (2020) Decellularized extracellular matrix-based bio-ink with enhanced 3D printability and mechanical properties. Biofabrication. https:// doi.org/10.1088/1758-5090/ab5d80

Kong JS, Huang X, Choi YJ, Yi HG, Kang J, Kim S, Kim J, Lee H, Rim YA, Ju JH, Chung WK, Woolf CJ, Jang J, Cho DW (2021) promoting long-term cultivation of motor neurons for 3D neuromuscular junction formation of $3 \mathrm{D}$ in vitro using central-nervous-tissue-derived bioink. Adv Healthc Mater. https://doi.org/10. 1002/adhm.202100581

Kupfer ME, Lin WH, Ravikumar V, Qiu K, Wang L, Gao L, Bhuiyan DB, Lenz M, Ai J, Mahutga RR, Townsend DW, Zhang J, McAlpine MC, Tolkacheva EG, Ogle BM (2020) In situ expansion, differentiation, and electromechanical coupling of human cardiac muscle in a 3D bioprinted, chambered organoid. Circ Res. https://doi.org/10.1161/CIRCRESAHA.119.316155

Langer EM, Allen-Petersen BL, King SM, Kendsersky ND, Turnidge MA, Kuziel GM, Riggers R, Samatham R, Amery TS, Jacques SL, Sheppard BC, Korkola JE, Muschler JL, Thibault G, Chang YH, Gray JW, Presnell SC, Nguyen DG, Sears RC (2019) Modeling tumor phenotypes in vitro with three-dimensional bioprinting. Cell Rep 26(3):608-623.e6. https://doi.org/10.1016/j.celrep. 2018.12.090

Larkindale J, Yang W, Hogan PF, Simon CJ, Zhang Y, Jain A, HabeebLouks EM, Kennedy A, Cwik VA (2014) Cost of illness for neuromuscular diseases in the United States. Muscle Nerve 49(3):431-438. https://doi.org/10.1002/mus.23942

Lee H, Han W, Kim H, Ha DH, Jang J, Kim BS, Cho DW (2017) Development of liver decellularized extracellular matrix bioink for three-dimensional cell printing-based liver tissue engineering. 
Biomacromol 18(4):1229-1237. https://doi.org/10.1021/acs.biomac.6b01908

Lee A, Hudson AR, Shiwarski DJ, Tashman JW, Hinton TJ, Yerneni S, Bliley JM, Campbell PG, Feinberg AW (2019) 3D bioprinting of collagen to rebuild components of the human heart. Science 365(6452):482-487. https://doi.org/10.1126/science.aav9051

Lelièvre SA, Kwok T, Chittiboyina S (2017) Architecture in 3D cell culture: an essential feature for in vitro toxicology. Toxicol in Vitro 45:287-295. https://doi.org/10.1016/j.tiv.2017.03.012

Li J, Liu Y, Zhang Y, Yao B, Enhejirigala B, Li Z, Song W, Wang Y, Duan X, Yuan X, Fu X, Huang S (2021) Biophysical and biochemical cues of biomaterials guide mesenchymal stem cell behaviors. Front Cell Dev Biol. https://doi.org/10.3389/fcell. 2021.640388

Lind JU, Yadid M, Perkins I, O'Connor BB, Eweje F, Chantre CO, Hemphill MA, Yuan H, Campbell PH, Vlassak JJ, Parker KK (2017) Cardiac microphysiological devices with flexible thinfilm sensors for higher-throughput drug screening. Lab Chip 17(21):3692-3703. https://doi.org/10.1039/c7lc00740j

Lindquist JA, Mertens PR (2018) Cold shock proteins: from cellular mechanisms to pathophysiology and disease. Cell Commun Signal 16:63. https://doi.org/10.1186/s12964-018-0274-6

Liu J, Song H, Zhang L, Xu H, Zhao X (2010) Self-assembly-peptide hydrogels as tissue-engineering scaffolds for three-dimensional culture of chondrocytes in vitro. Macromol Biosci 10(10):11641170. https://doi.org/10.1002/mabi.200900450

Liu J, Miller K, Ma X, Dewan S, Lawrence N, Whang G, Chung P, McCulloch AD, Chen S (2020) Direct 3D bioprinting of cardiac micro-tissues mimicking native myocardium. Biomaterials. https://doi.org/10.1016/j.biomaterials.2020.120204

Lu Y, Mapili G, Suhali G, Chen S, Roy K (2006) A digital micromirror device-based system for the microfabrication of complex, spatially patterned tissue engineering scaffolds. J Biomed Mater Res Part A 77(2):396-405. https://doi.org/10.1002/jbm.a.30601

Ma X, Qu X, Zhu W, Li Y-S, Yuan S, Zhang H, Liu J, Wang P, Lai CSE, Zanella F, Feng G-S, Sheikh F, Chien S, Chen S (2016) Deterministically patterned biomimetic human iPSC-derived hepatic model via rapid 3D bioprinting. Proc Nat Acad Sci 113(8):2206-2211. https://doi.org/10.1073/pnas.1524510113

Ma X, Liu J, Zhu W, Tang M, Lawrence N, Yu C, Gou M, Chen S (2018a) 3D bioprinting of functional tissue models for personalized drug screening and in vitro disease modeling. Adv Drug Deliv Rev 132:235-251. https://doi.org/10.1016/j.addr.2018.06. 011

Ma X, Yu C, Wang P, Xu W, Wan X, Lai CSE, Liu J, Koroleva-Maharajh A, Chen S (2018b) Rapid 3D bioprinting of decellularized extracellular matrix with regionally varied mechanical properties and biomimetic microarchitecture. Biomaterials 185:310-321. https://doi.org/10.1016/j.biomaterials.2018.09.026

Ma X, Dewan S, Liu J, Tang M, Miller KL, Yu C, Lawrence N, McCulloch AD, Chen S (2019) 3D printed micro-scale force gauge arrays to improve human cardiac tissue maturation and enable high throughput drug testing. Acta Biomater 95:319-327. https://doi.org/10.1016/j.actbio.2018.12.026

Madorran E, Stožer A, Bevc S, Maver U (2020) In vitro toxicity model: upgrades to bridge the gap between preclinical and clinical research. Bosn J Basic Med Sci 20(2):157-168. https://doi.org/ 10.17305/bjbms.2019.4378

Maffioletti SM, Sarcar S, Henderson ABH, Mannhardt I, Pinton L, Moyle LA, Steele-Stallard H, Cappellari O, Wells KE, Ferrari G, Mitchell JS, Tyzack GE, Kotiadis VN, Khedr M, Ragazzi M, Wang W, Duchen MR, Patani R, Zammit PS, Tedesco FS et al (2018) Three-dimensional human iPSC-derived artificial skeletal muscles model muscular dystrophies and enable multilineage tissue engineering. Cell Rep 23(3):899-908. https://doi.org/10. 1016/j.celrep.2018.03.091
Mao Q, Wang Y, Li Y, Juengpanich S, Li W, Chen M, Yin J, Fu J, Cai $X(2020)$ Fabrication of liver microtissue with liver decellularized extracellular matrix (dECM) bioink by digital light processing (DLP) bioprinting. Mater Sci Eng C. https://doi.org/10. 1016/j.msec.2020.110625

Massa S, Sakr MA, Seo J, Bandaru P, Arneri A, Bersini S, ZareEelanjegh E, Jalilian E, Cha BH, Antona S, Enrico A, Gao Y, Hassan S, Acevedo JP, Dokmeci MR, Zhang YS, Khademhosseini A, Shin SR (2017) Bioprinted 3D vascularized tissue model for drug toxicity analysis. Biomicrofluidics. https://doi.org/10. $1063 / 1.4994708$

Mathur A, Ma Z, Loskill P, Jeeawoody S, Healy KE (2016) In vitro cardiac tissue models: Current status and future prospects. Adv Drug Deliv Rev 96:203-213. https://doi.org/10.1016/j.addr.2015. 09.011

Mazzocchi A, Devarasetty M, Huntwork R, Soker S, Skardal A (2019) Optimization of collagen type I-hyaluronan hybrid bioink for 3D bioprinted liver microenvironments. Biofabrication. https://doi. org/10.1088/1758-5090/aae543

McCain ML, Sheehy SP, Grosberg A, Goss JA, Parker KK (2013) Recapitulating maladaptive, multiscale remodeling of failing myocardium on a chip. Proc Natl Acad Sci USA 110(24):9770 9775. https://doi.org/10.1073/pnas.1304913110

Meng F, Meyer CM, Joung D, Vallera DA, McAlpine MC, Panoskaltsis-Mortari A (2019) 3D Bioprinted in vitro metastatic models via reconstruction of tumor microenvironments. Adv Mater. https://doi.org/10.1002/adma.201806899

Miller KL, Xiang Y, Yu C, Pustelnik J, Wu J, Ma X, Matsui T, Imahashi K, Chen S (2021) Rapid 3D BioPrinting of a human iPSCderived cardiac micro-tissue for high-throughput drug testing. Organs-on-a-Chip 3:100007. https://doi.org/10.1016/j.ooc.2021. 100007

Mobaraki M, Ghaffari M, Yazdanpanah A, Luo Y, Mills DK (2020) Bioinks and bioprinting: a focused review. Bioprinting. https:// doi.org/10.1016/j.bprint.2020.e00080

Mondal A, Gebeyehu A, Miranda M, Bahadur D, Patel N, Ramakrishnan S, Rishi AK, Singh M (2019) Characterization and printability of sodium alginate-gelatin hydrogel for bioprinting NSCLC co-culture. Sci Rep. https://doi.org/10.1038/ s41598-019-55034-9

Mondrinos MJ, Alisafaei F, Yi AY, Ahmadzadeh H, Lee I, Blundell C, Seo J, Osborn M, Jeon T-J, Kim SM, Shenoy VB, Huh D (2021) Surface-directed engineering of tissue anisotropy in microphysiological models of musculoskeletal tissue. Sci Adv. https://doi. org/10.1126/sciadv.abe9446

Mouser VHM, Levato R, Mensinga A, Dhert WJA, Gawlitta D, Malda J (2020) Bio-ink development for three-dimensional bioprinting of hetero-cellular cartilage constructs. Connect Tissue Res 61(2):137-151. https://doi.org/10.1080/03008207.2018.1553960

Mueller D, Krämer L, Hoffmann E, Klein S, Noor F (2014) 3D organotypic HepaRG cultures as in vitro model for acute and repeated dose toxicity studies. Toxicol in Vitro 28(1):104-112. https://doi. org/10.1016/j.tiv.2013.06.024

Nam KH, Smith AST, Lone S, Kwon S, Kim DH (2015) Biomimetic 3D tissue models for advanced high-throughput drug screening. J Lab Autom 20(3):201-215. https://doi.org/10.1177/2211068214 557813

Osaki T, Uzel SGM, Kamm RD (2018) Microphysiological 3D model of amyotrophic lateral sclerosis (ALS) from human iPS-derived muscle cells and optogenetic motor neurons. Sci Adv. https://doi. org $/ 10.1126 /$ sciadv.aat5847

Ouyang L, Highley CB, Rodell CB, Sun W, Burdick JA (2016) 3D printing of shear-thinning hyaluronic acid hydrogels with secondary cross-linking. ACS Biomater Sci Eng 2(10):1743-1751. https://doi.org/10.1021/acsbiomaterials.6b00158 
Patel P (2016) The path to printed body parts. ACS Cent Sci 2(9):581583. https://doi.org/10.1021/acscentsci.6b00269

Polini A, del Mercato LL, Barra A, Zhang YS, Calabi F, Gigli G (2019) Towards the development of human immune-system-on-a-chip platforms. Drug Discov Today 24(2):517-525. https://doi.org/ 10.1016/j.drudis.2018.10.003

Przysinda A, Feng W, Li G (2020) Diversity of organism-wide and organ-specific endothelial cells. Curr Cardio Rep. https://doi.org/ 10.1007/s11886-020-1275-9

Ramaiahgari SC, den Braver MW, Herpers B, Terpstra V, Commandeur JNM, van de Water B, Price LS (2014) A 3D in vitro model of differentiated HepG2 cell spheroids with improved liver-like properties for repeated dose high-throughput toxicity studies. Arch Toxicol 88(5):1083-1095. https://doi.org/10.1007/ s00204-014-1215-9

Ronaldson-Bouchard K, Ma SP, Yeager K, Chen T, Song LJ, Sirabella D, Morikawa K, Teles D, Yazawa M, Vunjak-Novakovic G (2018) Advanced maturation of human cardiac tissue grown from pluripotent stem cells. Nature 556(7700):239-243. https:// doi.org/10.1038/s41586-018-0016-3

Ruskowitz ER, Deforest CA (2019) Proteome-wide analysis of cellular response to ultraviolet light for biomaterial synthesis and modification. ACS Biomater Sci Eng 5(5):2111-2116. https:// doi.org/10.1021/acsbiomaterials.9b00177

Saha SK, Wang D, Nguyen VH, Chang Y, Oakdale JS, Chen S-C (2019) Scalable submicrometer additive manufacturing. Science 366(6461):105-109. https://doi.org/10.1126/science.aax8760

Santhanam N, Kumanchik L, Guo X, Sommerhage F, Cai Y, Jackson M, Martin C, Saad G, McAleer CW, Wang Y, Lavado A, Long CJ, Hickman JJ (2018) Stem cell derived phenotypic human neuromuscular junction model for dose response evaluation of therapeutics. Biomaterials 166:64-78. https://doi.org/10.1016/j. biomaterials.2018.02.047

Sanz B, Sanchez AA, Tangey B, Gilmore K, Yue Z, Liu X, Wallace G (2021) Light cross-linkable marine collagen for coaxial printing of a 3D model of neuromuscular junction formation. Biomedicines 9(1):1-19. https://doi.org/10.3390/biomedicines9010016

Sasserath T, Rumsey JW, McAleer CW, Bridges LR, Long CJ, Elbrecht D, Schuler F, Roth A, Bertinetti-LaPatki C, Shuler ML, Hickman JJ (2020) Differential monocyte actuation in a three-organ functional innate immune system-on-a-chip. Adv Sci. https://doi. org/10.1002/advs.202000323

Schmidt K, Berg J, Roehrs V, Kurreck J, Al-Zeer MA (2020) 3D-bioprinted HepaRG cultures as a model for testing long term aflatoxin B1 toxicity in vitro. Toxicol Rep 7:1578-1587. https://doi. org/10.1016/j.toxrep.2020.11.003

Shao L, Gao Q, Xie C, Fu J, Xiang M, He Y (2020) Directly coaxial 3D bioprinting of large-scale vascularized tissue constructs. Biofabrication. https://doi.org/10.1088/1758-5090/ab7e76

Shrestha J, Razavi Bazaz S, Aboulkheyr Es H, Yaghobian Azari D, Thierry B, Ebrahimi Warkiani M, Ghadiri M (2020) Lung-on-achip: the future of respiratory disease models and pharmacological studies. Crit Rev Biotechnol 40(2):213-230. https://doi.org/ 10.1080/07388551.2019.1710458

Shusteff M, Browar AEM, Kelly BE, Henriksson J, Weisgraber TH, Panas RM, Fang NX, Spadaccini CM (2017) One-step volumetric additive manufacturing of complex polymer structures. Sci Adv 3:12. https://doi.org/10.1126/sciadv.aao5496

Skylar-Scott MA, Uzel SGM, Nam LL, Ahrens JH, Truby RL, Damaraju S, Lewis JA (2019) Biomanufacturing of organ-specific tissues with high cellular density and embedded vascular channels. Sci Adv. https://doi.org/10.1126/sciadv.aaw2459

Solomon EA, Rooney AM, Rodriguez AM, Micheva-Viteva S, Bashir R, Iyer R, Harris JF (2021) Neuromuscular junction model optimized for electrical platforms. Tissue Eng Part C Methods 27(4):242-252. https://doi.org/10.1089/ten.tec.2020.0292
Sung JH (2021) Multi-organ-on-a-chip for pharmacokinetics and toxicokinetic study of drugs. Expert Opin Drug Metab Toxicol 17(8):969-986. https://doi.org/10.1080/17425255.2021.1908996

Tang M, Xie Q, Gimple RC, Zhong Z, Tam T, Tian J, Kidwell RL, Wu Q, Prager BC, Qiu Z, Yu A, Zhu Z, Mesci P, Jing H, Schimelman J, Wang P, Lee D, Lorenzini MH, Dixit D, Rich JN et al (2020) Three-dimensional bioprinted glioblastoma microenvironments model cellular dependencies and immune interactions. Cell Res 30(10):833-853. https://doi.org/10.1038/s41422-020-0338-1

Tang M, Tiwari SK, Agrawal K, Tan M, Dang J, Tam T, Tian J, Wan X, Schimelman J, You S, Xia Q, Rana TM, Chen S (2021) Rapid 3D bioprinting of glioblastoma model mimicking native biophysical heterogeneity. Small. https://doi.org/10.1002/smll.202006050

Tariq I, Ali MY, Janga H, Ali S, Amin MU, Ambreen G, Ali U, Pinnapireddy SR, Schäfer J, Schulte LN, Bakowsky U (2020) Downregulation of MDR 1 gene contributes to tyrosine kinase inhibitor induce apoptosis and reduction in tumor metastasis: a gravity to space investigation. Int J Pharm. https://doi.org/10.1016/j.ijpha rm.2020.119993

Taubenberger AV, Bray LJ, Haller B, Shaposhnykov A, Binner M, Freudenberg U, Guck J, Werner C (2016) 3D extracellular matrix interactions modulate tumour cell growth, invasion and angiogenesis in engineered tumour microenvironments. Acta Biomater 36:73-85. https://doi.org/10.1016/j.actbio.2016.03.017

Tiburcy M, Meyer T, Liaw NY, Zimmermann WH (2020) Generation of engineered human myocardium in a multi-well format. STAR Protoc. https://doi.org/10.1016/j.xpro.2020.100032

Underhill GH, Khetani SR (2018) Advances in engineered human liver platforms for drug metabolism studies. Drug Metab Dispos 46(11):1626-1637. https://doi.org/10.1124/dmd.118.083295

Utama RH, Tan VTG, Tjandra KC, Sexton A, Nguyen DHT, O’Mahony AP, Du EY, Tian P, Ribeiro JCC, Kavallaris M, Gooding JJ (2021) A covalently crosslinked ink for multimaterials drop-ondemand 3D bioprinting of 3D cell cultures. Macromol Biosci. https://doi.org/10.1002/mabi.202100125

van Hoorick J, Tytgat L, Dobos A, Ottevaere H, van Erps J, Thienpont H, Ovsianikov A, Dubruel P, van Vlierberghe S (2019) (Photo-) crosslinkable gelatin derivatives for biofabrication applications. Acta Biomater 97:46-73. https://doi.org/10.1016/j.actbio.2019. 07.035

Vannozzi L, Yasa IC, Ceylan H, Menciassi A, Ricotti L, Sitti M (2018) Self-folded hydrogel tubes for implantable muscular tissue scaffolds. Macromol Biosci. https://doi.org/10.1002/mabi.201700377

Vargas-Valderrama A, Messina A, Mitjavila-Garcia MT, Guenou H (2020) The endothelium, a key actor in organ development and hPSC-derived organoid vascularization. J Biomed Sci. https:// doi.org/10.1186/s12929-020-00661-y

Veldhuizen J, Migrino RQ, Nikkhah M (2019) Three-dimensional microengineered models of human cardiac diseases. J Biol Eng. https://doi.org/10.1186/s13036-019-0155-6

Vila OF, Uzel SGM, Ma SP, Williams D, Pak J, Kamm RD, VunjakNovakovic G (2019) Quantification of human neuromuscular function through optogenetics. Theranostics 9(5):1232-1246. https://doi.org/10.7150/thno.25735

Vila OF, Chavez M, Ma SP, Yeager K, Zholudeva LV, Colón-Mercado JM, Qu Y, Nash TR, Lai C, Feliciano CM, Carter M, Kamm RD, Judge LM, Conklin BR, Ward ME, McDevitt TC, VunjakNovakovic G (2021) Bioengineered optogenetic model of human neuromuscular junction. Biomaterials. https://doi.org/10.1016/j. biomaterials.2021.121033

Vinson BT, Phamduy TB, Shipman J, Riggs B, Strong AL, Sklare SC, Murfee WL, Burow ME, Bunnell BA, Huang Y, Chrisey DB (2017) Laser direct-write based fabrication of a spatially-defined, biomimetic construct as a potential model for breast cancer cell invasion into adipose tissue. Biofabrication. https://doi.org/10. 1088/1758-5090/aa6bad 
Wang G, McCain ML, Yang L, He A, Pasqualini FS, Agarwal A, Yuan H, Jiang D, Zhang D, Zangi L, Geva J, Roberts AE, Ma Q, Ding J, Chen J, Wang DZ, Li K, Wang J, Wanders RJA, Pu WT et al (2014) Modeling the mitochondrial cardiomyopathy of Barth syndrome with induced pluripotent stem cell and heart-on-chip technologies. Nat Med 20(6):616-623. https://doi.org/10.1038/ nm.3545

Wang X, Zhang X, Dai X, Wang X, Li X, Diao J, Xu T (2018) Tumorlike lung cancer model based on 3D bioprinting. 3 Biotech. https://doi.org/10.1007/s13205-018-1519-1

Wang Z, Wang L, Li T, Liu S, Guo B, Huang W, Wu Y (2021) 3D bioprinting in cardiac tissue engineering. Theranostics 11(16):79487969. https://doi.org/10.7150/THNO.61621

Williams K, Liang T, Massé S, Khan S, Hatkar R, Keller G, Nanthakumar K, Nunes SS (2021) A 3-D human model of complex cardiac arrhythmias. Acta Biomater 132:149-161. https://doi. org/10.1016/j.actbio.2021.03.004

Xing JF, Zheng ML, Duan XM (2015) Two-photon polymerization microfabrication of hydrogels: an advanced 3D printing technology for tissue engineering and drug delivery. Chem Soc Rev 44(15):5031-5039. https://doi.org/10.1039/c5cs00278h

Xu F, Celli J, Rizvi I, Moon S, Hasan T, Demirci U (2011) A threedimensional in vitro ovarian cancer coculture model using a high-throughput cell patterning platform. Biotechnol J 6(2):204212. https://doi.org/10.1002/biot.201000340

Xu W, Zhang X, Yang P, Långvik O, Wang X, Zhang Y, Cheng F, Österberg M, Willför S, Xu C (2019) Surface engineered biomimetic inks based on UV cross-linkable wood biopolymers for 3D printing. ACS Appl Mater Interfaces 11(13):12389-12400. https://doi.org/10.1021/acsami.9b03442

Yang R, Li G, Zhuang C, Yu P, Ye T, Zhang Y, Shang P, Huang J, Cai M, Wang L, Cui W, Deng L (2021) Gradient bimetallic ion-based hydrogels for tissue microstructure reconstruction of tendon-tobone insertion. Sci Adv 7:26. https://doi.org/10.1126/sciadv. $\operatorname{abg} 3816$

You S, Guan J, Alido J, Hwang HH, Yu R, Kwe L, Su H, Chen S (2020) Mitigating scattering effects in light-based three-dimensional printing using machine learning. J Manuf Sci Eng Trans ASME. https://doi.org/10.1115/1.4046986

Yu C, Ma X, Zhu W, Wang P, Miller KL, Stupin J, Koroleva-Maharajh A, Hairabedian A, Chen S (2019) Scanningless and continuous 3D bioprinting of human tissues with decellularized extracellular matrix. Biomaterials 194:1-13. https://doi.org/10.1016/j.bioma terials.2018.12.009

Yu C, Miller KL, Schimelman J, Wang P, Zhu W, Ma X, Tang M, You S, Lakshmipathy D, He F, Chen S (2020a) A sequential 3D bioprinting and orthogonal bioconjugation approach for precision tissue engineering. Biomaterials. https://doi.org/10.1016/j.bioma terials.2020.120294

Yu C, Schimelman J, Wang P, Miller KL, Ma X, You S, Guan J, Sun B, Zhu W, Chen S (2020b) Photopolymerizable biomaterials and light-based 3D printing strategies for biomedical applications. Chem Rev 120(19):10695-10743. https://doi.org/10.1021/acs. chemrev.9b00810

Zhang W, Chen S (2011) Femtosecond laser nanofabrication of hydrogel biomaterial. MRS Bull 36(12):1028-1033. https://doi.org/10. 1557/mrs.2011.275

Zhang AP, Qu X, Soman P, Hribar KC, Lee JW, Chen S, He S (2012) Rapid fabrication of complex 3D extracellular microenvironments by dynamic optical projection stereolithography. Adv Mater 24(31):4266-4270. https://doi.org/10.1002/adma.20120 2024

Zhong Z, Balayan A, Tian J, Xiang Y, Hwang HH, Wu X, Deng X, Schimelman J, Sun Y, Ma C, dos Santos A, You S, Tang M, Yao E, Shi X, Steinmetz NF, Deng SX, Chen S (2021a) Bioprinting of dual ECM scaffolds encapsulating limbal stem/progenitor cells in active and quiescent statuses. Biofabrication. https://doi.org/ 10.1088/1758-5090/ac1992

Zhong Z, Deng X, Wang P, Yu C, Kiratitanaporn W, Wu X, Schimelman J, Tang M, Balayan A, Yao E, Tian J, Chen L, Zhang K, Chen S (2021b) Rapid bioprinting of conjunctival stem cell micro-constructs for subconjunctival ocular injection. Biomaterials. https://doi.org/10.1016/j.biomaterials.2020.120462

Zhu W, Holmes B, Glazer RI, Zhang LG (2016) 3D printed nanocomposite matrix for the study of breast cancer bone metastasis. Nanomed Nanotechnol Biol Med 12(1):69-79. https://doi.org/ 10.1016/j.nano.2015.09.010

Zhu W, Qu X, Zhu J, Ma X, Patel S, Liu J, Wang P, Lai CSE, Gou M, Xu Y, Zhang K, Chen S (2017) Direct 3D bioprinting of prevascularized tissue constructs with complex microarchitecture. Biomaterials 124:106-115. https://doi.org/10.1016/j.bioma terials.2017.01.042

Zuppinger C (2019) 3D cardiac cell culture: a critical review of current technologies and applications. Front Cardiovasc Med. https://doi. org/10.3389/fcvm.2019.00087

Publisher's Note Springer Nature remains neutral with regard to jurisdictional claims in published maps and institutional affiliations. 